\title{
A simple model of production and trade in an oligopolistic market: back to basics
}

\author{
SOMDEB LAHIRI \\ School of Petroleum Management \\ PD Petroleum University \\ Raisan, Gandhinagar, 382 007, Gujarat, India
}

(Received: July 15, 2016)

\begin{abstract}
We provide a two good model of oligopolistic production and trade with one good being commodity money. There is the usual demand function of the consumers for the produced good that producer-sellers face. Each seller is a budget constrained preference maximizer and derives utility (or satisfaction) from consuming bundles comprising commodity money and the produced good. We define a competitive equilibrium strategy profile and a Cournotian equilibrium and show that under our assumptions both exist. We further show that at a competitive equilibrium strategy profile, each seller maximizes profits given his own consumption of the produced good and the price of the produced good, the latter being determined by the inverse demand function. Similarly we show that at a Cournotian the sellers are at a Cournot equilibrium given their own consumption of the produced good. Assuming sufficient differentiability of the cost functions we show that at a competitive equilibrium each seller either sets price equal to marginal cost or exhausts his capacity of production; at a Cournotian equilibrium each seller either sets marginal revenue equal to marginal cost or exhausts his capacity of production. We also study the evolution of Cournotian strategies as the sellers and buyers are replicated. As the number of buyers and sellers go to infinity any sequence of interior symmetric Cournotian equilibrium strategies admits a convergent subsequence, which converges to an interior symmetric competitive equilibrium strategy. In a final section we discuss the Bertrand Edgeworth price setting game and show that a Bertrand Edgeworth equilibrium must be a derived from a competitive equilibrium price. Here we show that if at a symmetric competitive equilibrium, the sellers consume positive quantities of the produced good then the competitive equilibrium cannot be a Bertrand Edgeworth equilibrium. Thus, if at all symmetric competitive equilibria the sellers consume positive amounts of the produced good, then a Bertrand Edgeworth equilibrium simply does not exist.
\end{abstract}

Mathematics Subject Classifications (2015). 91A40, 91B24

Keywords. oligopoly, competitive equilibrium, Cournotian equilibrium, existence, asymptotic convergence 


\section{Introduction}

The conventional model of oligopoly with production, whether it be that of Cournot or that of Bertrand is one where there are profit maximizing firms facing a Marshallian demand or inverse demand curve. In this context a Cournot-Nash equilibrium is a list of quantities supplied by each firm such that given what other firms are supplying each firm chooses its profit maximizing output if it adheres to the recommendations in the list. This is the model that is used to study oligopoly in the industrial organization literature right from the beginning and it continues to be the framework in which oligopoly with production is investigated even now. A reasonably up-to-date survey of the literature relating to the Cournot solution in this simple model and its myriad properties can be found in [18].

There is an alternative literature concerning oligopolistic markets that has grown in recent times. In this model there is no production but only trade. Some traders own a non-monetary good and others are initially endowed with commodity money. In this game each owner of the non-monetary good chooses to supply a quantity of the good. The total amount of the non-monetary good supplied determines its market price. Each seller chooses a quantity to sell in such a way that the resulting consumption bundle consisting of the nonmonetary good that he retains with him and the amount of money that he earns by selling the good maximizes his utility. A Nash equilibrium of this game played by all the sellers of the non-monetary is what is expected to materialize in this trading economy. Contributions in this line of research are available in $[7][10][11]$ and a few others.

The motivation behind this paper is slightly different. Consider the market for fish. As in the simple oligopoly model there is an inverse demand function for fish which the sellers in the market face. However do the sellers of fish harvest only that amount which they sell on the market? Don't they themselves consume some of the fish that they catch? The literature on oligopolistic models for a homogeneous good with production is silent on this issue. There are other more "newsworthy" markets, for which the same question applies. Take for instance the international market for "crude oil" which is usually modeled as an oligopoly (see [5]). In this market there are a few producers of crude oil and the rest are consumers. The producers of crude oil earn profits by selling crude oil on the market. At the same time they themselves consume crude oil for their daily needs and other industrial activity. A case in point is Saudi Arabia, a major producer of crude oil that suddenly decided to reduce oil exports and domestic consumption of the same, simply because oil is an exhaustible resource and accelerated depletion of the resource could one day lead to dire consequences for them. If they did not care about domestic consumption of oil, then they would manage oil supplies in the same way that they were doing till date, factoring in as always the oligopolistic nature of the market and the possibilities of cartel formation. Clearly, that does not seem to be the case. Sellers also consume the good that they sell in the market. This is amply taken care of in oligopolistic models of pure exchange. In oligopolistic markets with 
production this phenomena is not given importance at all.

To take care of such possibilities, general equilibrium models of oligopolistic markets have been suggested, most notably that of Gabszewicz and Vial in [8]. This model is analogous to the Arrow-Debreu general equilibrium model with production, with utility maximizing consumers behaving like price takers and firms transferring their outputs to the consumers. Each consumer gets the fraction of the total output of a firm in proportion to its share in the firm. With these as initial endowments, the consumers trade to an Arrow-Debreu equilibrium. It is assumed that there is a unique Arrow-Debreu price vector corresponding to an aggregate output produced by firms. This is the inverse demand function faced by the firms, who subsequently use this inverse demand function to arrive ata Cournot equilibrium. Elegant as the story might be, this model is beleagured with existence of equilibrium problems and the problem of the equilibrium (if it exists at all!) depending on the choice of numeraire. The work in [8] has proved very useful in providing a host of examples (and some counter examples) relating to the behavior of an economy. However, it has been found wanting in providing a solid theoretical foundation for further research in the area. There is an alternative model of production and trade with all consumers and firms behaving strategically due to Dubey and Shubik in [6]. The interactions between agents take place over two periods and is based on bids and offers that each individual agent makes. The model considered by in [6] is closer in spirit to the work done in [16][14][15] and not to the models we consider here.

With this is mind we provide a two good model of oligopolistic production and trade as a compromise between the classical partial equilibrium model of profit maximizing firms facing an inverse demand function and the full blown general equilibrium model in [8]. In our model there is commodity money which is consumed and at the same time used as a means of payment. At the same time there is a good for which there is the usual demand function of the consumers that producer-sellers face. Each seller is a budget constrained preference maximizer and derives utility (or satisfaction) from consuming bundles comprising commodity money and the produced good. The producers incur costs to produce the latter type of good part of which they sell to the consumers and part of which they consume themselves. The profits measured in units of commodity money that the sellers earn by selling the produced good on the market is consumed by the sellers. While the revenue that a seller earns is the payment that he receives from selling the produced good in the market to the consumers, the cost of producing the produced good is based on the total amount produced by him part of which he sells on the market and part of which he himself consumes. No seller sells the produced good to any other seller; sellers only sell to buyers whose inverse demand function they are aware of.

Our assumptions on the inverse demand function and the utility functions of the sellers is quite standard and in the initial stages quite general as well. We assume each seller faces a finite capacity constraint, beyond which he cannot 
produce any amount at all. This may or may not be a standard assumption, but it is definitely very realistic. Throughout the paper we invoke a Positivity Assumption on the cost functions and the inverse demand function which appears to be of a technical nature but is satisfied by almost all kinds of cost functions and inverse demand functions. We define a competitive equilibrium strategy profile and a Cournotian equilibrium and show that under our assumptions both exist. We further show that at a competitive equilibrium strategy profile, each seller maximizes profits given his own consumption of the produced good and the price of the produced good, the latter being determined by the inverse demand function. Similarly we show that at a Cournotian the sellers are at a Cournot equilibrium given their own consumption of the produced good. Assuming sufficient differentiability of the cost functions we show that at a competitive equilibrium each seller either sets price equal to marginal cost or exhausts his capacity of production; at a Cournotian equilibrium each seller either sets marginal revenue equal to marginal cost or exhausts his capacity of production. All this follows from and in spite of sellers being assumed to be budget constrained utility maximizers.

Subsequently we study the evolution of Cournotian strategies as the sellers and buyers are replicated. Similar analysis in a trading economy has been carried out in [10]. Novshek in [13] studies the asymptotic convergence of Counot equilibrium strategies to competitive equilibrium strategies in the classical model of oligopoly. In this section we assume twice continuous differentiability of the cost and utility functions and also assume that they are "well behaved". Each seller is a replica of any other and a similar assumption is made for the buyers. Under these conditions it is natural to look for symmetric competitive equilibrium strategies and symmetric Cournotian equilibrium strategies. Further these strategies are interior symmetric equilibrium strategies when they satisfy their respective marginal conditions. The possibility of there being more than one interior symmetric competitive equilibrium strategies is noted. Further given any two interior symmetric competitive equilibrium strategies, at one the sellers consume more of both goods and are all better off than at the other. At the better strategy they sell less on the market though they produce more of the produced good. As the number of buyers and sellers go to infinity any sequence of interior symmetric Cournotian equilibrium strategies admits a convergent subsequence, which converges to an interior symmetric competitive equilibrium strategy. If there is a unique interior symmetric competitive equilibrium strategy, then the sequence itself converges to it.

In a final section of this paper we turn to the price setting game due to Bertrand and Edgeworth but incorporating the fact that our sellers are utility maximizers and not profit maximizers as is assumed in the existing literature. To keep matters simple we assume all sellers are identical in every respect and the cost function is linear with a capacity constraint as before. In the absence of capacity constraints it is well known that price competition game (known as the Bertrand competition) among profit maximizing sellers results in price being set equal to unit costs. However when we have capacity constraints which "bite" 
as happens for instance if we assume the Positivity Assumption, then there is no way in which market prices can come down to unit costs without leaving some of the buyers unsatisfied. In this kind of a game it is usually assumed that there is some kind of a rationing rule by which selling in the presence of excess demand is met. There are two types of rationing rules that are prominent in the literature: proportional and surplus maximizing. Beckmann in [2] discusses the first rule and Levitan and Shubik in [12] discuss the second. What we use here is a version of the surplus maximizing rationing rule, since that appears to be the natural way a decentralized market would function.

As in the case of a profit-maximizing oligopoly, there is a problem concerning the existence of pure strategy equilibrium in our model. [19] provides a lucid discussion of problems relating to existence of pure strategy equilibrium and conditions under which existence of such equilibrium may be possible in the profit maximizing model. Tasnadi in [17] shows that for profit maximizing sellers with zero unit cost of production, a Bertrand Edgeworth equilibrium exists if the price elasticity of demand is greater than one whenever aggregate demand does not exceed the total output capacity of the sellers.

We show here that pure strategy equilibrium of the Bertrand Edgeworth game must of necessity be derived from a competitive equilibrium price. If at a symmetric competitive equilibrium, the sellers consume positive quantities of the produced good then the competitive equilibrium cannot be a Bertrand Edgeworth equilibrium. Thus, if at all symmetric competitive equilibria the sellers consume positive amounts of the produced good, then a Bertrand Edgeworth equilibrium simply does not exist.

Some of the results reported here echo the results in oligopoly theory when sellers are profit maximizers and some do not. Often additional conditions require to be imposed in our model so as to obtain the desired results. These conditions sometimes reduce to familiar conditions if we assume that sellers do not care about their own consumption of the produced good. Thus our approach may be viewed as an attempt towards generalization of the model in which oligopoly theory is usually discussed.

\section{The Model}

In this section we develop a two good model in which there is commodity money and another good that is produced using money. There are $H>1$ sellers of the produced commodity and we use $h=1, \ldots, H$ to denote a seller who also produces the produced good from commodity money. The $\mathrm{H}$ sellers sell the produced good in a market and the inverse demand function for the good is given by $p: \mathbb{R}_{+} \rightarrow \mathbb{R}_{+}$. Here $\mathrm{R}$ denotes the set of real numbers, $\mathbb{R}_{+}$denotes the set of nonnegative real numbers and $\mathbb{R}_{++}$denotes the set of strictly positive real numbers. We use $\mathbb{R}_{+}^{*}$ to denote the set $\mathbb{R}_{+} \cup\{+\infty\}$ of extended real numbers.

Each seller $h$ has a cost function $c^{h}: \mathbb{R}_{+} \rightarrow \mathbb{R}_{+}^{*}$ such that to produce $\eta$ units of the produced good seller $h$ has to use $c^{h}(\eta)$ units of money. The preferences 
of seller $h$ are given by a utility function $u^{h}: \mathbb{R}_{+}^{2} \rightarrow \mathbb{R}$. The utility derived by seller $\mathrm{h}$ from consuming $\xi \geq 0$ units of the produced good and $\alpha \geq 0$ units of money is given by $u^{h}(\alpha, \xi)$.

If the total amount of the produced good sold on the market by all the sellers is $y$ and if seller $h$ sells an amount $y^{h}$ on the market, then the amount of money earned by seller $h$ is $p(y) y^{h}$. If in addition seller $h$ wants to consume $x^{h}$ units of the produced good, then the total amount of the produced good that seller $h$ needs to produce is $x^{h}+y^{h}$ and for that he needs to spend $c^{h}\left(x^{h}+y^{h}\right)$ units of commodity money. In such a situation the commodity bundle consumed by seller $h$ is $\left(p(y) y^{h}-c^{h}\left(x^{h}+y^{h}\right), x^{h}\right)$ if $p(y) y^{h}-c^{h}\left(x^{h}+y^{h}\right) \geq 0$. He thus derives a utility of $u^{h}\left(p(y) y^{h}-c^{h}\left(x^{h}+y^{h}\right), x^{h}\right)$ if $p(y) y^{h}-c^{h}\left(x^{h}+y^{h}\right) \geq 0$.

In what follows we assume that each seller $h$ has a productive capacity $\bar{x}^{h}>0$, such that $c^{h}(\xi) \in \mathbb{R}_{+}$if and only if $0 \leq \xi \leq \bar{x}^{h}$. Thus, $c^{h}(\xi)=+\infty$ if $\xi>\bar{x}^{h}$. In the rest of the paper when we use $c^{h}$ we will (unless otherwise mentioned) assume that it is a function from the closed interval $\left[0, \bar{x}^{h}\right]$ to $\mathbb{R}_{+}$.

Example:For all $h=1, \ldots, H, c^{h}=0$ if $0 \leq \xi \leq \bar{x}^{h}$.

This is the simple pure trade model, where seller $h$ is endowed with $\bar{x}^{h}>0$ of the produced good.

A strategy for player ' $h$ ' is an ordered pair $\left(x^{h}, y^{h}\right) \in \mathbb{R}_{+}^{2}$ with $x^{h}+y^{h} \leq \bar{x}^{h}$. Here $x^{h}$ denotes the amount of the produced good that $h$ consumes and $y^{h}$ denotes the amount of the produced good that $h$ sells on the market. Let $S^{h}$ denote the strategy set of seller $h$.

A strategy profile is a list $<\left(x^{h}, y^{h}\right) \mid h=1, \ldots, H>$ such that $\left(x^{h}, y^{h}\right)$ is a strategy for seller $h$. Let $S=\prod_{h=1}^{H} S^{h}$ denote the set of strategy profiles.

For all $\alpha \geq 0$, define the function $R_{\alpha}: \mathbb{R}_{+} \rightarrow \mathbb{R}_{+}$by $R_{\alpha}(y)=y p(y+\alpha)$ for $y \geq 0$.

We now make the following assumptions about the inverse demand function $p: \mathbb{R}_{+} \rightarrow \mathbb{R}_{+}(i) p(0)>0$.

(ii) $p$ is continuous .

(iii) $p$ is twice continuously differentiable with $p^{\prime} \leq 0$ on the set $\left\{y \in \mathbb{R}_{++} \mid p(y)>\right.$ $0\}$ and $\lim _{y \rightarrow 0} y p^{\prime}(y)=0$.

(iv) For all $\alpha \geq 0$, the function $R_{\alpha}: \mathbb{R}_{+} \rightarrow \mathbb{R}_{+}$is continuous, concave on $y \in \mathbb{R}_{+} \mid p(y+\alpha)>0$ and has non-positive second derivative on $\left\{y \in \mathbb{R}_{+} \mid y+\alpha>\right.$ 0 and $p(y+\alpha)>0\}$.

About $c^{h}, h=1, \ldots, H$, we make the following assumptions:

$(i) c^{h}(0)=0$.

(ii) $c^{h}$ is continuous, strictly increasing and convex.

About $u^{h}, h=1, \ldots, H$, we make the following assumptions:

$(i) u^{h}$ is continuous, concave and weakly increasing (i.e. $\alpha, \xi, \alpha^{\prime}, \xi^{\prime} \in R_{+}, \alpha^{\prime}>$ $\alpha, \xi^{\prime}>\xi$, implies $\left.u^{h}\left(\alpha^{\prime}, \xi^{\prime}\right)>u^{h}(\alpha, \xi)\right)$; 
(ii) $\alpha, \xi, \alpha^{\prime}, \xi^{\prime} \in R_{+}, u^{h}\left(\alpha^{\prime}, \xi^{\prime}\right)>u^{h}(0,0), u^{h}(\alpha, \xi)>u^{h}(0,0), \alpha^{\prime} \geq \alpha$ and $\xi^{\prime} \geq \xi$ with at least one of the last two inequalities being strict implies $u^{h}\left(\alpha^{\prime}, \xi^{\prime}\right)>$ $u^{h}(\alpha, \xi)$.

Given a strategy profile $<\left(x^{* h}, y^{* h}\right) \mid h=1, \ldots, H>$ and a seller $g \in 1, \ldots, H$, let $B^{g}\left(<\left(x^{* h}, y^{* h}\right) \mid h=1, \ldots, H>\right)=\left\{\left(x^{g}, y^{g}\right) \in S^{g} \mid y^{g} p^{*}{ }^{g} c^{g}\left(x^{g}+y^{g}\right) \geq 0\right\}$ be the competitive budget set of seller $g$, where $p^{*}=p\left(\sum_{h=1}^{H} y^{* h}\right)$.

A strategy profile $<\left(x^{* h}, y^{* h}\right) \mid h=1, \ldots, H>$ is said to be a competitive equilibrium strategy profile if for all $h=1, \ldots, H,\left(x^{* h}, y^{* h}\right)$ maximizes $u\left(p^{*} y^{h}-c^{h}\left(x^{h}+y^{h}\right), x^{h}\right)$ subject to $\left(x^{h}, y^{h}\right) \in B^{h}\left(<\left(x^{* g}, y^{* g}\right) \mid g=1, \ldots, H>\right)$. In this case, $p\left(\sum_{h=1}^{H} y^{* h}\right)$ is said to be the competitive equilibrium price vector.

Seller $h$ is said to be a competitive profit maximizer at the strategy profile $<\left(x^{h}, y^{h}\right) \mid h=1, \ldots, H>$ if $p\left(\sum_{h=1}^{H} y^{h}\right) y^{h \smile} c^{h}\left(x^{h}+y^{h}\right) \geq p\left(\sum_{h=1}^{H} y^{h}\right) y^{\prime h} c^{h}(x+$ $\left.y^{\prime h}\right)$ for all $y^{\prime h} \in\left[0, \bar{x}^{h}-x^{h}\right]$.

Given a strategy profile $<\left(x^{g}, y^{g}\right) \mid g=1, \ldots, H>$ and $h \in 1, \ldots, H$, let $\mathfrak{B}^{h}\left(<\left(x^{g}, y^{g}\right) \mid g=1, \ldots, H>\right)=\left\{\left(x^{\prime h}, y^{\prime h}\right) \in S^{h} \mid R_{\alpha}\left(y^{\prime h}\right)-c^{h}\left(x^{\prime h}+y^{\prime h}\right) \geq 0\right.$, where $\left.\alpha=\sum_{g \neq h} y^{g}\right\} \cdot \mathfrak{B}^{h}\left(<\left(x^{g}, y^{g}\right) \mid g=1, \ldots, H>\right)$ is called the Cournotian budget set of $h$.

A strategy profile $<\left(x^{c g}, y^{c g}\right) \mid g=1, \ldots, H>$ is said to be a Cournotian equilibrium if for all $h=1, \ldots, H$, for all $h=1, \ldots, H,\left(x^{c h}, y^{c h}\right)$ solves

Maximize $u^{h}\left(y^{\prime h} p\left(y^{\prime h}+\sum_{m} i n g \neq h y^{c g}\right)-c^{h}\left(x^{\prime h}+y^{\prime h}\right), x^{\prime h}\right)$

subject to $\left(x^{\prime h}, y^{\prime h}\right) \in \mathfrak{B}^{h}\left(<\left(x^{c g}, y^{c g}\right) \mid g=1, \ldots, H>\right)$.

A strategy profile $<\left(x^{h}, y^{h}\right) \mid h=1, \ldots, H>$ is said to be a Cournot equilibrium if for all $h=1, \ldots, H$ and for all $y^{\prime h} \in\left[0, \bar{x}^{h}-x^{h}\right]: p\left(\sum_{h=1}^{H} y^{h}\right) y^{h \smile} c^{h}\left(x^{h}+\right.$ $\left.y^{h}\right) \geq p\left(\sum_{g \neq h} y^{g}+y^{\prime h}\right) y^{\prime h} c^{h}\left(x^{h}+y^{\prime h}\right)$.

\section{Existence of competitive equilibrium and com- petitive profit mazimization:}

In this section we will adjust the proof due to Arrow and Debreu (1954) to show that a competitive equilibrium exists in our context.We begin with an assumption.

Positivity Assumption:For all $h=1, \ldots, H$, there exists a strategy $\left(\hat{x}^{h}, \hat{y}^{h}\right)$ such that $p\left(\sum_{g=1}^{H} \bar{x}^{g}\right) \hat{y}^{h}-c^{h}\left(\hat{x}^{h}+\hat{y}^{h}\right)>0$

It is easy to see that the Positivity Assumption implies $\left[0, \sum_{h=1}^{H} \bar{x}^{h}\right] \subset\{y \in$ 
$\left.\mathbb{R}_{+} \mid p(y)>0\right\}$.

This is not an unreasonable assumption given that we assume that cost functions are convex and there are no (long run) fixed costs. If $p\left(\sum_{g=1}^{H} \bar{x}^{g}\right)$ is greater than the limit as output goes to zero of marginal costs, then it is easy to see that there are two positive numbers $\xi$ and $\eta$ with $\xi>\eta$, both very small such that $p\left(\sum_{g=1}^{H} \bar{x}^{g}\right) \eta-c^{h}(\xi)>0$. In this case the Positivity Assumption clearly holds. Thus if $p\left(\sum_{g=1}^{H} \bar{x}^{g}\right)>0$ and the limit of marginal costs as output goes to zero is zero (e.g. quadratic cost functions) then the Positivity Assumption holds.

A crucial step in the proof of this result is the next lemma. In what follows in this section and also in the subsequent section we will use the notions of correspondence, upper-semicontinuous correspondence, lower-semi continuous correspondence and continuous correspondence. For definitions of the same, please see [4].

Lemma 1 Suppose the economy satisfies the Positivity Assumption. Then, for all $h=1, \ldots, H$, the competitive budget correspondence $B^{h}: S \rightarrow \rightarrow \mathbb{R}_{+}^{2}$ is nonempty valued, convex valued, compact valued and continuous.

Proof: Let $<\left(x^{* h}, y^{* h}\right) \mid h=1, \ldots, H>\in S$ and $p^{*}=p\left(\sum_{h=1}^{H} y^{* h}\right)$. It is easy to see that given our assumptions $B^{g}\left(<\left(x^{* h}, y^{* h}\right) \mid h=1, \ldots, H>\right)=$ $\left\{\left(x^{g}, y^{g}\right) \in S^{g} \mid y^{g} p^{*} c^{g}\left(x^{g}+y^{g}\right) \geq 0\right\}$ is convex-valued and compact valued. In fact boundedness of $B^{g}\left(<\left(x^{* h}, y^{* h}\right) \mid h=1, \ldots, H>\right)$ follows from the fact that if $B^{g}\left(<\left(x^{* h}, y^{* h}\right) \mid h=1, \ldots, H>\right) \subset\left\{\left(x^{g}, y^{g}\right) \in \mathbb{R}_{+}^{2} \mid x^{g}+y^{g} \leq \bar{x}^{g}\right\}$ and $B^{g}\left(<\left(x^{* h}, y^{* h}\right) \mid h=1, \ldots, H>\right)$ is closed because we have made adequate continuity assumptions on the functions $p($.$) and c($.$) . Further, B^{g}\left(<\left(x^{* h}, y^{* h}\right) \mid h=\right.$ $1, \ldots, H>)$ is non-empty since $(0,0)$ belongs to it.

Let $<<\left(x^{* h(k)}, y^{* h(k)}\right)|h=1, \ldots, H>| k \in \mathbb{N}>$ be a sequence of strategies in $S$ converging to $<\left(x^{* h}, y^{* h}\right) \mid h=1, \ldots, H>\in S$. Let $<<\left(x^{h(k)}, y^{h(k)}\right) \mid h=$ $1, \ldots, H>\mid k \in \mathbb{N}>$ be a sequence with $\left(x^{g(k)}, y^{g(k)}\right) \in B^{g}\left(<\left(x^{* h(k)}, y^{* h(k)}\right) \mid h=\right.$ $1, \ldots, H>)$ for all $k \in \mathbb{N}$. Suppose $<\left(x^{g(k)}, y^{g(k)}\right) \mid k \in \mathbb{N}>$ converges to $\left(x^{g}, y^{g}\right)$. Then by our assumptions on the function $p($.$) , the sequence <y^{g(k)} p\left(\sum_{h=1}^{H} y^{(} *\right.$ $h(k))) \mid k \in \mathbb{N}>$ converges to $y^{g} p\left(\sum_{h=1}^{H} y^{* h}\right)$ and by our assumptions on the function $c\left(\right.$.), the sequence $\left\langle c^{g}\left(x^{g(k)}+y^{g(k)}\right)\right| k \in \mathbb{N}>$ converges to $c^{g}\left(x^{g}+y^{g}\right)$. Since $y^{g(k)} p\left(\sum_{h=1}^{H} y^{* h(k)}\right)-c^{g}\left(x^{g(k)}+y^{g(k)}\right) \geq 0$ for all $k \in \mathbb{N}$, it must be the case that $y^{g} p\left(\sum_{h=1}^{H} y^{* h}\right)-c^{g}\left(x^{g}+y^{g}\right) \geq 0$. Thus $\left(x^{g}, y^{g}\right) \in B^{g}\left(<\left(x^{* h}, y^{* h}\right) \mid h=\right.$ $1, \ldots, H>)$. Thus the correspondence $B^{g}$ is upper semi-continuous.

Now let us show that $B^{g}$ is lower semi-continuous. Let $<<\left(x^{* h(k)}, y^{* h(k)}\right) \mid h=$ $1, \ldots, H>\mid k \in \mathbb{N}>$ be a sequence of strategies in $S$ converging to $<\left(x^{* h}, y^{* h}\right) \mid h=$ $1, \ldots, H>\in S$ and suppose $\left(x^{g}, y^{g}\right) \in B^{g}\left(<\left(x^{* h}, y^{* h}\right) \mid h=1, \ldots, H>\right)$.

By the Positivity Assumption, and since the function $p($.$) is non-increasing,$ 
$p\left(\sum_{h=1}^{H} \tilde{y}^{h}\right) \hat{y}^{g}-c^{g}\left(\hat{x}^{g}+\hat{y}^{g}\right)>0$, whenever $<\left(\tilde{x}^{h}, \tilde{y}^{h}\right) \mid h=1, \ldots, H>\in S$.

Since $S$ is a compact set and since the function $p($.$) is continuous and non-$ decreasing, there exists $\delta>0$ such that $p\left(\sum_{h=1}^{H} \tilde{y}^{h}\right) \hat{y}^{g}-c^{g}\left(\hat{x}^{g}+\hat{y}^{g}\right) \geq \delta$ for all $<\left(\tilde{x}^{h}, \tilde{y}^{h}\right) \mid h=1, \ldots, H>\in S$.

Thus, $p\left(\sum_{h=1}^{H} y^{* h}\right) \hat{y}^{g}-c^{g}\left(\hat{x}^{g}+\hat{y}^{g}\right) \geq \delta$ and $p\left(\sum_{h=1}^{H} y^{* h(k)}\right) \hat{y}^{g}-c^{g}\left(\hat{x}^{g}+\hat{y}^{g}\right) \geq \delta$ for all $k \in \mathbb{N}$.

Now, $p\left(\sum_{h=1}^{H} y^{* h}\right) y^{g}-c^{g}\left(x^{g}+y^{g}\right) \geq 0$.

Let $M=1$. Since $<<\left(x^{* h(k)}, y^{* h(k)}\right)|h=1, \ldots, H>| k \in \mathbb{N}>$ is a sequence of strategies in $S$ converging to $<\left(x^{* h}, y^{* h}\right) \mid h=1, \ldots, H>\in S$, there exists a positive integer $N_{1}$ such that for all $k \geq N_{1}, p\left(\sum_{h=1}^{H} y^{* h(k)}\right) y^{g}-c^{g}\left(x^{g}+y^{g}\right) \geq-\delta$.

Having defined the positive integer $N_{M}$ for a positive integer $M$, let $N_{M+1}>$ $N_{M}$ be a positive integer such that for all $k \geq N_{M+1}, p\left(\sum_{h=1}^{H} y^{* h(k)}\right) y^{g}-c^{g}\left(x^{g}+\right.$ $\left.y^{g}\right) \geq-\delta / M+1$. This is possible since $<<\left(x^{* h(k)}, y^{* h(k)}\right)|h=1, \ldots, H>| k \in$ $\mathbb{N}>$ is a sequence of strategies in $S$ converging to $<\left(x^{* h}, y^{* h}\right) \mid h=1, \ldots, H>\in S$.

Let $x^{g(k)}=\hat{x}^{g}, y^{g(k)}=\hat{y}^{g}$ for $1 \leq k<N_{1}, x^{g(k)}=\hat{x}^{g} / M+1+M x^{g} / M+1$ and $y^{g(k)}=\hat{y}^{g} / M+1+M y^{g} / M+1$ for all $N_{M} \leq k<N_{M+1}, M \in \mathbb{N}$.

Then for $1 \leq k<N_{1}$,

$p\left(\sum_{h=1}^{H} y^{* h(k)}\right) y^{g(k)}-c^{g}\left(x^{g(k)}+y^{g(k)}\right)=p\left(\sum_{h=1}^{H} y^{* h(k)}\right) \hat{y}^{g}-c^{g}\left(\hat{x}^{g}+\hat{y}^{g}\right) \geq \delta>0$.

For $M \in \mathbb{N}$ and $N_{M} \leq k<N_{M+1}$,

$$
\begin{aligned}
& p\left(\sum_{h=1}^{H} y^{* h(k)}\right) y^{g(k)}-c^{g}\left(x^{g(k)}+y^{g(k)}\right)= \\
& p\left(\sum_{h=1}^{H} y^{* h(k)}\right)\left[\frac{\hat{y}^{g}}{M+1}+\frac{M y^{g}}{M+1}\right]-c^{g}\left(\frac{\hat{x}^{g}}{M+1}+\frac{M x^{g}}{M+1}+\frac{\hat{y}^{g}}{M+1}+\frac{M y^{g}}{M+1}\right) \geq \\
& \frac{1}{M+1}\left[p\left(\sum_{h=1}^{H} y^{* h(k)}\right) \hat{y}^{g}-c^{g}\left(\hat{x}^{g}+\hat{y}^{g}\right)\right]+\frac{M}{M+1}\left[p\left(\sum_{h=1}^{H} y^{* h(k)}\right) y^{g}-c^{g}\left(x^{g}+y^{g}\right)\right]= \\
& \frac{1}{M+1} \delta-\frac{M}{M+1} \frac{\delta}{M}=0 .
\end{aligned}
$$

Thus, $\left(x^{g(k)}, y^{g(k)}\right) \in B^{g}\left(<\left(x^{* h(k)}, y^{* h(k)}\right) \mid h=1, \ldots, H>\right)$ for all $k \in \mathbb{N}$.

Further, $\lim _{k \rightarrow \infty} x^{g(k)}=x^{g}$ and $\lim _{k \rightarrow \infty} y^{g(k)}=y^{g}$. 
Thus the competitive budget correspondence $B^{g}$ is lower semi-continuous. Combined with the upper semi-continuity of $B^{g}$ we get that the competitive budget correspondence $B^{g}$ is continuous.Q.E.D.

Our proof of the following theorem (and subsequently that of Theorem 3) mimics the proof of the existence of Nash equilibrium for games in strategic form due to Geanakoplos in [9].

Theorem 1 Suppose the economy satisfies the Positivity Assumption. Then a competitive equilibrium strategy profile exists. Further, if there are two sellers $h_{1}$ and $h_{2}$ who are identical in every respect (i.e. $u^{h_{1}}=u^{h_{2}}, c^{h_{1}}=c^{h_{2}}$ and $\left.\bar{x}^{h_{1}}=\bar{x}^{h_{2}}\right)$, then there exists a competitive equilibrium strategy profile with the strategies of $h_{1}$ and $h_{2}$ being identical. The latter statement can be generalized to any number of identical sellers.

Proof: $B^{h}$ is non-empty valued, convex valued, compact valued and continuous by Lemma 1 . Also each $S^{h}$ is a non-empty, compact and convex subset of $\mathbb{R}_{+}^{2}$.

Given $<\left(x^{g}, y^{g}\right) \mid g=1, \ldots, H>\in S$, and $h \in\{1, \ldots, H\}$, consider the problem

Maximize $u^{h}\left(y^{\prime h} p\left(\sum_{g=1}^{H} y^{g}\right)^{\smile} c^{h}\left(x^{\prime h}+y^{\prime h}\right), x^{\prime h}\right)^{\smile}\left(x^{\prime}{ }^{\smile} x^{h}\right)^{2} \smile\left(y^{\prime h}\left\llcorner y^{h}\right)^{2}\right.$

subject to $\left(x^{\prime h}, y^{\prime h}\right) \in B^{h}\left(<\left(x^{g}, y^{g}\right) \mid g=1, \ldots, H>\right)$.

Let $p=p\left(\sum_{g=1}^{H} y^{g}\right)$. By the Positivity assumption, there exists a strategy $\left(\hat{x}^{h}, \hat{y}^{h}\right)$ such that $p \hat{y}^{h}-c^{h}\left(\hat{x}^{h}+\hat{y}^{h}\right)>0$. Thus, $p>0$ and $\hat{y}^{h}>0$. Clearly there exists $\epsilon>0$ such that $\hat{y}^{h}-\epsilon>0$ and $p\left(\hat{y}^{h}-\epsilon\right)-c^{g}\left(\hat{x}^{h}+\hat{y}^{h}\right)>$ 0 . Thus $\left(\hat{x}^{h}+\epsilon, \hat{y}^{h}-\epsilon\right) \in B^{h}\left(<\left(x^{g}, y^{g}\right) \mid g=1, \ldots, H>\right) \cap \mathbb{R}_{++}^{2}$. Thus, $u^{h}\left(p\left(\hat{y}^{h}-\epsilon\right)^{\smile} c^{h}\left(\hat{x}^{h}+\hat{y}^{h}\right), \hat{x}^{h}\right)>u^{h}(0,0)$.

By the continuity and strict concavity of the objective function on the constraint set and the compactness of the constraint set, the above problem has a unique solution. Let $f^{h}: S \rightarrow S^{h}$ be the function such that for $<\left(x^{g}, y^{g}\right) \mid g=$ $1, \ldots, H>\in S, f^{h}\left(<\left(x^{g}, y^{g}\right) \mid g=1, \ldots, H>\right)$ is the unique solution to the above optimization problem. Let us show that $f^{h}$ is continuous.

Let $<<\left(x^{g(k)}, y^{g(k)}\right)|g=1, \ldots, H>| k \in \mathbb{N}>$ be a sequence in $\mathrm{S}$ converging to $\left\langle\left(x^{g}, y^{g}\right)\right| g=1, \ldots, H>$. Since $S$ is compact (and therefore closed) $<\left(x^{g}, y^{g}\right) \mid g=1, \ldots, H>$ belongs to $S$. Let $f^{h}\left(<\left(x^{g(k)}, y^{g(k)}\right) \mid g=1, \ldots, H>\right.$ )$=\left(\bar{x}^{h(k)}, \bar{y}^{h(k)}\right)$ for $k \in \mathbb{N}$ and let $f^{h}\left(<\left(x^{g}, y^{g}\right) \mid g=1, \ldots, H>\right)=\left(\bar{x}^{h}, \bar{y}^{h}\right)$. We have to show that $\lim _{k \rightarrow \infty}\left(\bar{x}^{h(k)}, \bar{y}^{h(k)}\right)=\left(\bar{x}^{h}, \bar{y}^{h}\right)$.

Suppose not. Then there exists a $\delta>0$ and a subsequence of

$$
<\left(\bar{x}^{h(k)}, \bar{y}^{h(k)}\right) \mid k \in \mathbb{N}>
$$


such that the distance of every term of this subsequence from $\left(\bar{x}^{h}, \bar{y}^{h}\right)$ is at least $\delta$. Since the set $S^{h}$ is compact and this subsequence lies in $S^{h}$ it admits a further subsequence which converges to some $\left(\bar{x}^{\prime} h, \bar{y}^{\prime} h\right)$ in $S^{h}$. Without loss of generality (and to save on some notation) suppose this subsequence which converges to $\left(\bar{x}^{\prime} h, \bar{y}^{\prime} h\right)$ is the original sequence $<\left(\bar{x}^{h(k)}, \bar{y}^{h(k)}\right) \mid k \in N>$. By the upper semi-continuity of $B^{h},\left(\bar{x}^{\prime h}, \bar{y}^{\prime h}\right) \in B^{h}\left(<\left(x^{g}, y^{g}\right) \mid g=1, \ldots, H>\right)$. Further since the distance of each term of the sequence from $\left(\bar{x}^{h}, \bar{y}^{h}\right)$ is at least $\delta$, the distance of $\left(\bar{x}^{\prime h}, \bar{y}^{\prime} h\right)$ from $\left(\bar{x}^{h}, \bar{y}^{h}\right)$ must be at least $\delta$.

Let $p^{(k)}=\sum_{g=1}^{H} y^{g(k)}$ and $p=\sum_{g=1}^{H} y^{g}$. Clearly $p>0$ and $p^{(k)}>0$ for all $k \in \mathbb{N}$ and by the continuity of the function $p(),. \lim _{k \rightarrow \infty} p^{(k)}=p$.

Let $\left(x^{\prime h}, y^{\prime h}\right) \in B^{h}\left(<\left(x^{g}, y^{g}\right) \mid g=1, \ldots, H>\right)$. Then by the lower semicontinuity of $B^{h}$, there exists a sequence $<\left(x^{\prime} h(k), y^{\prime} h(k)\right) \mid k \in \mathbb{N}>$ such that for all $k \in \mathbb{N},\left(x^{\prime h(k)}, y^{\prime h(k)}\right) \in B^{h}\left(<\left(x^{g(k)}, y^{g(k)}\right) \mid g=1, \ldots, H>\right)$ and

$$
\lim _{k \rightarrow \infty}\left(x^{\prime h(k)}, y^{\prime h(k)}\right)=\left(x^{\prime h}, y^{\prime h}\right)
$$

$\left(x^{\prime h(k)}, y^{\prime h(k)}\right) \in B^{h}\left(<\left(x^{g(k)}, y^{g(k)}\right) \mid g=1, \ldots, H>\right)$ and $f^{h}\left(<\left(x^{g(k)}, y^{g(k)}\right) \mid g=\right.$ $1, \ldots, H>)=\left(\bar{x}^{h(k)}, \bar{y}^{h(k)}\right)$ implies $u^{h}\left(\bar{y}^{h(k)} p^{(k)} \backsim c^{h}\left(\bar{x}^{h(k)}+\bar{y}^{h(k)}\right), \bar{x}^{h(k)}\right) \geq$ $u^{h}\left(y^{\prime h(k)} p^{(k)}{ }^{\iota} c^{h}\left(x^{\prime h(k)}+y^{\prime h(k)}\right), x^{\prime h(k)}\right)$.

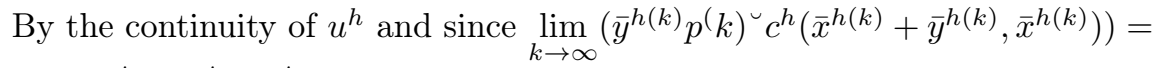

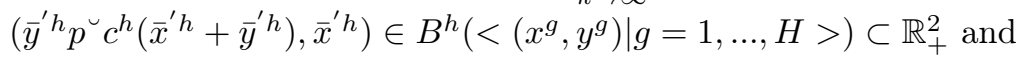

$$
\begin{aligned}
\lim _{k \rightarrow \infty}\left(y^{\prime h(k)} p^{(k)} \cup c^{h}\left(x^{\prime h(k)}+y^{\prime h(k)}\right), x^{\prime h(k)}\right) & = \\
& \left(y^{\prime h} p^{\smile} c^{h}\left(x^{\prime h}+y^{\prime h}\right), x^{\prime h}\right) \in B^{h}\left(<\left(x^{g}, y^{g}\right) \mid g=1, \ldots, H>\right) \subseteq \mathbb{R}_{+}^{2},
\end{aligned}
$$

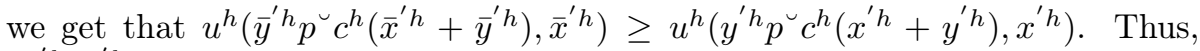
$\left(\bar{x}^{\prime} h, \bar{y}^{\prime} h\right)$ solves

Maximize $u^{h}\left(y^{\prime h} p^{\smile} c^{h}\left(x^{\prime h}+y^{\prime h}\right), x^{\prime h}\right)^{\smile}\left(x^{\prime h} x^{h}\right)^{2 \smile}\left(y^{\prime h} y^{h}\right)^{2}$

subject to $\left(x^{\prime h}, y^{\prime h}\right) \in B^{h}\left(<\left(x^{g}, y^{g}\right) \mid g=1, \ldots, H>\right)$.

Since, $\left(\bar{x}^{\prime} h, \bar{y}^{\prime} h\right) \neq\left(\bar{x}^{h}, \bar{y}^{h}\right)$ we arrive at a contradiction.

Thus, $f^{h}$ is continuous.

Consider the function $f: S \rightarrow S$, whose $h^{t h}$ component is $f^{h}$. $f$ is a continuous function from a compact convex subset of $\left(\mathbb{R}_{+}^{2}\right)^{H}$ to itself. Thus by Brouwer's fixed point theorem $f$ has a fixed point. 
Let $<\left(x^{* g}, y^{* g}\right) \mid g=1, \ldots, H>$ be a fixed point of $f$. Thus, for all $h \in$ $\{1, \ldots, H\},\left(x^{* h}, y^{* h}\right)$ solves

Maximize $u^{h}\left(y^{\prime h} p\left(\sum_{g=1}^{H} y^{* g}\right)^{\smile} c^{h}\left(x^{\prime h}+y^{\prime h}\right), x^{\prime h}\right)^{\smile}\left(x^{\prime} h^{\smile} x^{* h}\right)^{2}\left(y^{\prime}{ }^{\smile} y^{* h}\right)^{2}$

subject to $\left(x^{\prime h}, y^{\prime h}\right) \in B^{h}\left(<\left(x^{* g}, y^{* g}\right) \mid g=1, \ldots, H>\right)$.

Thus, for all $h=1, \ldots, H$,

$$
\begin{aligned}
& u^{h}\left(y^{* h} p\left(\sum_{g=1}^{H} y^{* g}\right)^{\smile} c^{h}\left(x^{* h}+y^{* h}\right), x^{* h}\right) \geq \\
& u^{h}\left(y^{\prime} h p\left(\sum_{g=1}^{H} y^{* g}\right)^{\smile} c^{h}\left(x^{\prime} h+y^{\prime} h\right), x^{\prime} h\right)^{\smile}\left(x^{\prime} h \cup x^{* h}\right)^{2 \smile}\left(y^{\prime} h \cup y^{* h}\right)^{2},
\end{aligned}
$$

whenever $\left(x^{\prime h}, y^{\prime h}\right) \in B^{h}\left(<\left(x^{* g}, y^{* g}\right) \mid g=1, \ldots, H>\right)$.

Let $p^{*}=p\left(\sum_{h=1}^{H} y^{* h}\right),\left(x^{\prime h}, y^{\prime h}\right) \in B^{h}\left(<\left(x^{* g}, y^{* g}\right) \mid g=1, \ldots, H>\right)$ and $\theta \in[0,1]$. Since $B^{h}\left(<\left(x^{* g}, y^{* g}\right) \mid g=1, \ldots, H>\right)$ is a convex set, $\left(x^{* h}, y^{* h}\right)+$ $\theta\left(x^{\prime h}-x^{* h}, y^{\prime h}-y^{* h}\right) \in B^{h}\left(<\left(x^{* g}, y^{* g}\right) \mid g=1, \ldots, H>\right)$ for all $\theta \in[0,1]$.

Thus, $u^{h}\left(y^{* h} p^{* \smile} c^{h}\left(x^{* h}+y^{* h}\right), x^{* h}\right) \geq u^{h}\left(\left(y^{* h}+\theta\left(y^{\prime h}-y^{* h}\right)\right) p^{*}-c^{h}\left(x^{* h}+\right.\right.$ $\left.y^{* h}+\theta\left(x^{\prime h}-x^{* h}+y^{\prime h}-y^{* h}\right), x^{* h}+\theta\left(x^{\prime h}-x^{* h}\right)\right)-\theta^{2}\left[\left(x^{\prime} h\right)^{2}+\left(y^{\prime h}\right)^{2}\right]$.

By the concavity of $u^{h}$ and the convexity of $c^{h}, u^{h}\left(\left(y^{* h}+\theta\left(y^{\prime h}-y^{* h}\right)\right) p *\right.$ $-c^{h}\left(x^{* h}+y^{* h}+\theta\left(x^{\prime} h-x^{* h}+y^{\prime h}-y^{* h}\right), x^{* h}+\theta\left(x^{\prime h}-x^{* h}\right)\right) \geq u^{h}\left(y^{* h} p^{*}-c^{h}\left(x^{* h}+\right.\right.$ $\left.\left.y^{* h}\right), x^{* h}\right)+\theta\left[u^{h}\left(y^{\prime} h p^{*}-c^{h}\left(x^{\prime} h+y^{\prime} h\right), x^{\prime h}\right)-u^{h}\left(y^{* h} p^{*}-c^{h}\left(x^{* h}+y^{* h}\right), x^{* h}\right)\right]$.

Thus, $u^{h}\left(y^{* h} p^{*} c^{h}\left(x^{* h}+y^{* h}\right), x^{* h}\right) \geq u^{h}\left(y^{* h} p^{*}-c^{h}\left(x^{* h}+y^{* h}\right), x^{* h}\right)+$ $\theta\left[u^{h}\left(y^{\prime h} p^{*}-c^{h}\left(x^{\prime h}+y^{\prime h}\right), x^{\prime h}\right)-u^{h}\left(y^{* h} p^{*}-c^{h}\left(x^{* h}+y^{* h}\right), x^{* h}\right)\right]-\theta^{2}\left[\left(x^{\prime h}\right)^{2}+\right.$ $\left.\left(y^{\prime} h\right)^{2}\right]$.

Hence $\theta^{2}\left[\left(x^{\prime h}\right)^{2}+\left(y^{\prime h}\right)^{2}\right] \geq \theta\left[u^{h}\left(y^{\prime h} p^{*}-c^{h}\left(x^{\prime h}+y^{\prime h}\right), x^{\prime h}\right)-u^{h}\left(y^{* h} p^{*}-\right.\right.$ $\left.\left.c^{h}\left(x^{* h}+y^{* h}\right), x^{* h}\right)\right]$ for all $\theta \in[0,1]$.

Dividing throughout by $\theta$ for $\theta \in(0,1]$, we get $\theta\left[\left(x^{\prime h}\right)^{2}+\left(y^{\prime h}\right)^{2}\right] \geq u^{h}\left(y^{\prime h} p^{*}-\right.$ $\left.c^{h}\left(x^{\prime h}+y^{\prime h}\right), x^{\prime h}\right)-u^{h}\left(y^{* h} p^{*}-c^{h}\left(x^{* h}+y^{* h}\right), x^{* h}\right)$ for all $\theta \in(0,1]$.

Letting $\theta$ tend to zero, we get $0 \geq u^{h}\left(y^{\prime h} p^{*}-c^{h}\left(x^{\prime h}+y^{\prime h}\right), x^{\prime h}\right)-u^{h}\left(y^{* h} p^{*}-\right.$ $\left.c^{h}\left(x^{* h}+y^{* h}\right), x^{* h}\right)$, i.e. $u^{h}\left(y^{* h} p^{*}-c^{h}\left(x^{* h}+y^{* h}\right), x^{* h}\right) \geq u^{h}\left(y^{\prime} h p^{*}-c^{h}\left(x^{\prime} h+\right.\right.$ $\left.\left.y^{\prime} h\right), x^{\prime} h\right)$.

Thus, $<\left(x^{* g}, y^{* g}\right) \mid g=1, . ., H>$ is a competitive equilibrium strategy profile.

To prove that there exists a competitive equilibrium strategy profile at which identical sellers choose identical strategies, suppose that sellers 1 and 
two are identical. Let $\left\langle\left(x^{g}, y^{g}\right)\right| g=1, \ldots, H>$ be a competitive equilibrium strategy profile and let $x=\frac{\left(x^{1}+x^{2}\right)}{2}, y=\frac{\left(y^{1}+y^{2}\right)}{2}$. Let $p=p\left(\sum_{g=1}^{H} y^{g}\right)=$ $p\left(y+y+\sum_{1 \neq g \neq 2} y^{g}\right)$. Let $\left(x^{\prime 1}, y^{\prime 1}\right) \in S^{1}$ with $p y^{\prime 1} c^{1}\left(x^{\prime 1}+y^{\prime 1}\right) \geq 0$. Then $u^{1}\left(p y^{1}-c^{1}\left(x^{1}+y^{1}\right), x^{1}\right) \geq u^{1}\left(p y^{\prime 1} c^{1}\left(x^{\prime 1}+y^{\prime}{ }^{1}\right), x^{\prime 1}\right)$ and since sellers 1 and 2 are identical, $u^{1}\left(p y^{2}-c^{1}\left(x^{2}+y^{2}\right), x^{2}\right) \geq u^{1}\left(p y^{\prime 1}{ }^{1} c^{1}\left(x^{\prime 1}+y^{\prime 1}\right), x^{\prime 1}\right)$. By the concavity of $u^{1}$ and convexity of $c^{1}, u^{1}\left(p y-c^{1}(x+y), x\right) \geq u^{1}\left(p y^{\prime 1} c^{1}\left(x^{\prime 1}+y^{\prime 1}\right), x^{\prime 1}\right)$.

Similarly, if $\left(x^{\prime 2}, y^{\prime 2}\right) \in S^{2}$ with $p y^{\prime 2}{ }^{2} c^{2}\left(x^{\prime 2}+y^{\prime 2}\right) \geq 0$, then $u^{2}\left(p y-c^{2}(x+\right.$ $y), x) \geq u^{2}\left(p y^{\prime 2}{ }^{2} c^{2}\left(x^{\prime 2}+y^{\prime 2}\right), x^{\prime 2}\right)$.

Thus the strategy profile, $<(x, y),(x, y),\left(x^{3}, y^{3}\right), \ldots,\left(x^{H}, y^{H}\right)>$ is a competitive equilibrium profile.

This argument can be easily generalized to any number of identical sellers. Q.E.D.

In order to show the continuity of the function $f$ we went through a derivation instead of appealing to the Maximum Theorem of Berge [3]. The reason for this is that for each $h$, the function $u^{h}$ is defined only on those pairs $\left(\xi p(\xi+\alpha)+c^{h}(\xi+\eta), \xi\right)$ which belong to $\mathbb{R}_{+}^{2}$. Hence were we to define a payoff function $V^{h}: S \rightarrow \mathbb{R}$ for h such that given $\alpha$ (the aggregate output of other sellers) $V^{h}$ evaluated at a strategy $(\xi, \eta)$ for $h$ is $u^{h}\left(\xi p(\xi+\alpha)+c^{h}(\xi+\eta), \xi\right)$ provided $\left(\xi p(\xi+\alpha)+c^{h}(\xi+\eta), \xi\right)$ belongs to $\mathbb{R}_{+}^{2}$ and something less than or equal to $u^{h}(0.0)$ otherwise, such a function $V^{h}$ would not be continuous on $S$. Hence the Maximum Theorem does not go through as stated, although its proof can be replicated in our context. That is what we have done.

Claim 1: Let $<\left(x^{* g}, y^{* g}\right) \mid g=1, \ldots, H>$ be a competitive equilibrium strategy profile and $p^{*}$ the competitive equilibrium price. Then $p^{*} y^{* g}>0$ for all $g=1, \ldots, H$.

Proof: Suppose $p^{*}=0$. Thus, $\sum_{g=1}^{H} y^{* g}>0$.Hence there exists a seller $h$, such that $y^{* h}>0$. Thus, $p^{*} y^{* h} c^{h}\left(x^{* h}+y^{* h}\right)<0$, since $x^{* h}+y^{* h}>0$ and $c^{h}$ is strictly increasing. This implies $\left(x^{* h}, y^{* h}\right) \notin B^{h}\left(<\left(x^{* g}, y^{* g}\right) \mid g=1, \ldots, H>\right)$, contradicting $<\left(x^{* g}, y^{* g}\right) \mid g=1, \ldots, H>$ is a competitive equilibrium strategy profile. Thus, $p^{*}>0$.

Hence suppose $y^{* h}=0$ for some seller $h$. Since it is required that $p^{*} y^{* h} u c^{h}\left(x^{* h}+\right.$ $\left.y^{* h}\right) \geq 0$, it must be the case that $x^{* h}=0$. By the Positivity Assumption $p^{*} \hat{y}^{h}-$ $c^{h}\left(\hat{x}^{h}+\hat{y}^{h}\right)>0$. Thus there exists $\epsilon>0$ such that $p^{*}\left(\hat{y}^{h}-\epsilon\right)-c^{h}\left(\hat{x}^{h}+\hat{y}^{h}\right)>0$ and $\hat{y}^{h}-\epsilon>0$. Thus $\left(\hat{y}^{h}-\epsilon, \hat{x}^{h}+\epsilon\right) \in B\left(<\left(x^{* g}, y^{* g}\right) \mid g=1, \ldots, H>\right) \cap \mathbb{R}_{++}^{2}$.

Thus, $u^{h}\left(p^{*} y^{* h}-c^{h}\left(x^{* h}+y^{* h}\right), x^{* h}\right) \geq u^{h}\left(p^{*}\left(\hat{y}^{h}-\epsilon\right)-c^{h}\left(\hat{x}^{h}+\hat{y}^{h}\right), \hat{x}^{h}+\epsilon\right)>$ $u^{h}(0,0)$, contradicting $\left(x^{* h}, y^{* h}\right)=(0.0)$. This proves the claim. Q.E.D.

In the next theorem we show that at competitive equilibrium strategy pro- 
files all sellers are profit maximizers.

THEOREM 2 Let $<\left(x^{* g}, y^{* g}\right) \mid g=1, \ldots, H>$ be a competitive equilibrium strategy profile. Then every seller is a competitive profit maximizer at this strategy profile.

Proof: Let $p^{*}$ denote $p\left(\sum_{g=1}^{H} y^{* g}\right)$ and consider seller h. We know that by the Positivity Assumption $p^{*} \hat{y}^{h}-c^{h}\left(\hat{x}^{h}+\hat{y}^{h}\right)>0$. Thus there exists $\epsilon>0$ such that $p^{*}\left(\hat{y}^{h}-\epsilon\right)-c^{h}\left(\hat{x}^{h}+\hat{y}^{h}\right)>0$ and $\hat{y}^{h}-\epsilon>0$. Thus $\left(\hat{y}^{h}-\epsilon, \hat{x}^{h}+\epsilon\right) \in B(<$ $\left.\left(x^{* g}, y^{* g}\right) \mid g=1, \ldots, H>\right) \cap \mathbb{R}_{++}^{2}$.

Thus, $u^{h}\left(p^{*} y^{* h}-c^{h}\left(x^{* h}+y^{* h}\right), x^{* h}\right) \geq u^{h}\left(p^{*}\left(\hat{y}^{h}-\epsilon\right)-c^{h}\left(\hat{x}^{h}+\hat{y}^{h}\right), \hat{x}^{h}+\epsilon\right)>$ $u^{h}(0,0)$.

Towards a contradiction suppose there exists $y^{h} \in\left[0, \bar{x}^{h}-x^{* h}\right]$, such that $p^{*} y^{h}-c^{h}\left(x^{* h}+y^{h}\right)>p^{*} y^{* h}-c^{h}\left(x^{* h}+y^{* h}\right)$.

Then by our hypothesis on preferences, $u^{h}\left(p^{*} y^{h}-c^{h}\left(x^{* h}+y^{h}\right), x^{* h}\right)>$ $u^{h}\left(p^{*} y^{* h}-c^{h}\left(x^{* h}+y^{* h}\right), x^{* h}\right)$, contradicting our assumption that $<\left(x^{* g}, y^{* g}\right) \mid g=$ $1, \ldots, H>$ is a competitive equilibrium strategy profile.

Thus every seller must be a competitive profit maximizer at this strategy profile. Q.E.D.

We propose the following property for cost functions:

Twice continuous differentiability of cost functions:For all $h=1, \ldots, H$, $c^{h}$ is twicecontinuously differentiable on $\left(0, \bar{x}^{h}\right)$ with $D c^{h}(\xi)>0$ and $D^{2} c^{h}(\xi) \geq$ 0 for all $\xi \in\left(0, \bar{x}^{h}\right)$.

$D c^{h}(\xi)$ is the marginal cost incurred by seller $h$ to produce $\xi$ units of the produced good and is often denoted $M c^{h}(\xi)$.

Proposition 1: Suppose twice continuous differentiability of cost functions. Let $<\left(x^{* g}, y^{* g}\right) \mid g=1, \ldots, H>$ be a competitive equilibrium strategy profile. Then for all $h=1, \ldots, H$, either $(a) p\left(\sum_{g=1}^{H} y^{* g}\right)=M c^{h}\left(x^{* h}+y^{* h}\right)$ or (b) $x^{* h}+y^{* h}=\bar{x}^{h}$.

Proof: Let $p^{*}=p\left(\sum_{g=1}^{H} y^{* g}\right)$ be the competitive equilibrium price. Consider seller $h$. By Theorem 2 and twice continuous differentiability of cost functions assumption, $p^{*}<M c^{h}\left(x^{* h}+y^{* h}\right)$ implies $y^{* h}=0$. By the Positivity Assumption it must be the case that $\left(x^{*}, y^{* h}\right) \in B^{h}\left(<\left(x^{* g}, y^{* g}\right) \mid g=\right.$ $1, \ldots, H>)$ and $u^{h}\left(p^{*} y^{* h}-c^{h}\left(x^{* h}+y^{* h}\right), x^{* h}\right)>u^{h}(0,0)$. Thus, $\left(p^{*} y^{* h}-\right.$ $\left.c^{h}\left(x^{* h}+y^{* h}\right), x^{* h}\right) \neq(0,0)$. Since $y^{* h}=0$, this is possible only if $x^{* h}>0$ and $c^{h}\left(x^{* h}+y^{* h}\right)=0$. But by twice continuous differentiability of cost functions 
assumption and $x^{* h}+y^{* h}>0$ it follows that $c^{h}\left(x^{* h}+y^{* h}\right)>0$, leading to a contradiction.

Towards a contradiction suppose that for some $h \in 1, \ldots, H, p^{*}>M c^{h}\left(x^{* h}+\right.$ $\left.y^{* h}\right)$ and yet $x^{* h}+y^{* h}<\bar{x}^{h}$. Choose $\epsilon>0$ such that $x^{* h}+y^{* h}+\epsilon<\bar{x}^{h}$ and $p^{*}>M c^{h}\left(x^{* h}+y^{* h}+\epsilon\right)$. This is clearly possible since the cost functions are continuously differentiable and $p^{*}>M c^{h}\left(x^{* h}+y^{* h}\right)$. Thus, $p^{*}\left(y^{* h}+\epsilon\right)^{\smile} c^{h}\left(x^{* h}+\right.$ $\left.y^{* h}+\epsilon\right)=p^{*} y^{* h \iota} c^{h}\left(x^{* h}+y^{* h}\right)+\epsilon\left(p^{*}-M c^{h}\left(x^{* h}+y^{* h}+\theta \epsilon\right)\right)$ for some $\theta \in(0,1)$. By the convexity of $\left.c^{h}, M c^{h}\left(x^{* h}+y^{* h}+\epsilon\right) \geq M c^{h}\left(x^{* h}+y^{* h}+\theta \epsilon\right)\right)$.

Thus, $p^{*} y^{* h \smile} c^{h}\left(x^{* h}+y^{* h}\right)+\epsilon\left(p^{*}-M c^{h}\left(x^{* h}+y^{* h}+\theta \epsilon\right)\right) \geq p^{*} y^{* h} c^{h}\left(x^{* h}+\right.$ $\left.y^{* h}\right)+\epsilon\left(p^{*}-M c^{h}\left(x^{* h}+y^{* h}+\epsilon\right)\right)>p^{*} y^{* h} c^{h}\left(x^{* h}+y^{* h}\right)$.

Hence, $p^{*}\left(y^{* h}+\epsilon\right)^{\smile} c^{h}\left(x^{* h}+y^{* h}+\epsilon\right)>p^{*} y^{* h} c^{h}\left(x^{* h}+y^{* h}\right)$.

But, then seller $h$ is not a competitive profit maximizer at $<\left(x^{* g}, y^{* g}\right) \mid g=$ $1, \ldots, H>$, contradicting Theorem 2. This proves the proposition. Q.E.D.

Note: If the cost function of a seller is linear i.e. the case of constant average/marginal cost, then it follows from the Positivity Assumption that price is greater than marginal cost. In such a situation it follows from Proposition 1, that the seller will exhaust his capacity of production.

\section{Cournotian and Cournot equilibrium strategy profiles:}

In this section we establish the existence of a Cournotian equilibrium if the Positivity Assumption is satisfied. Then we show that a Cournotian equilibrium is a Cournot equilibrium.

A crucial step for what follows is the next lemma.

Lemma 2 Suppose the economy satisfies the Positivity Assumption. Then, for all $h=1, \ldots, H$, the Cournotian budget correspondence $\mathfrak{B}^{h}: S \rightarrow \rightarrow \mathbb{R}_{+}^{2}$ is non-empty valued, convex valued, compact valued and continuous.

Proof:Non-empty valuedness follows from the fact that $(0,0)$ belongs to every Cournotian budget set. Convex valuedness follows from the concavity of $R_{\alpha}$ for all $\alpha \geq 0$ and the convexity of the cost functions. Every Cournotian budget set of a seller is bounded since it is a subset of his set of strategies which is a bounded subset of $\mathbb{R}_{+}^{2}$. Every Cournotian budget set of a seller is closed since the cost function of the seller as well as the functions $R_{\alpha}$ are all continuous and profits are required to be non-negative. 
From here the proof is very similar to the proof of the converging part in Lemma 1 and is therefore being omitted. Q.E.D.

Theorem 3 Suppose the economy satisfies the Positivity Assumption. Then a Cournotian equilibrium strategy profile exists. Further, if there are two sellers $h_{1}$ and $h_{2}$ who are identical in every respect $\left(i . e . u^{h_{1}}=u^{h_{2}}, c^{h_{1}}=c^{h_{2}}\right.$ and $\left.\bar{x}^{h_{1}}=\bar{x}^{h_{2}}\right)$, then there exists a Cournotian equilibrium strategy profile with the strategies of $h_{1}$ and $h_{2}$ being identical. The latter statement can be generalized to any number of identical sellers.

Proof: The proof of this is very similar to the proof of Theorem 1 and is therefore being omitted.

Claim 2: Let $<\left(x^{c g}, y^{c g}\right) \mid g=1, \ldots, H>$ be a Cournotian equilibrium strategy profile and let $p=p\left(\sum_{g=1}^{H} y^{c g}\right)$. Then $p y^{c g}>0$ for all $g=1, \ldots, H$.

Proof: The proof of this is very similar to the proof of Claim 1 and is therefore being omitted

The next result states that every Cournotian equilibrium strategy profile is also a Cournot equilibrium strategy profile.

THEOREM 4 Every Cournotian equilibrium strategy profile is a Cournot equilibrium strategy profile.

Proof: The proof of this is very similar to the proof of Theorem 2 and is therefore omitted.

For a seller $h$, a strategy $\left(x^{h}, y^{h}\right) \in S^{h}$ and $\alpha \geq 0$ with $y^{h}+\alpha>0$, let $M R_{\alpha}\left(y^{h}\right)=D R_{\alpha}\left(y^{h}\right)=y^{h} p^{\prime}\left(y^{h}+\alpha\right)+p\left(y^{h}+\alpha\right)$.

The following proposition is analogous to Proposition 1.

Proposition 2: Suppose twice continuous differentiability of cost functions. Let $<\left(x^{* g}, y^{* g}\right) \mid g=1, \ldots, H>$ be a Cournotian equilibrium strategy profile. For all $h=1, \ldots, H$, let $\alpha(h)=\sum_{g \neq h} y^{* g}$. Then for all $h=1, \ldots, H$, either $(a) M R_{\alpha(h)}\left(y^{* h}\right)=M c^{h}\left(x^{* h}+y^{* h}\right)$ or $(b) x^{* h}+y^{* h}=\bar{x}^{h}$.

Proof:The proof of this Proposition is very similar to the proof of Proposition 1 and is thus being omitted. 


\section{Limiting properties of Cournotian equilibria:}

In this section we investigate the behavior of Cournotian equilibria as the number of sellers in the market goes to infinity. For the sake of simplicity we will assume that all sellers in the market are identical- each seller is an identical copy of any other seller in the market.

We will also require to replicate the buyers' side of the market. Hence we postulate that the inverse demand function for the produced good in a market with $H$ buyers is a function $p^{(H)}: \mathbb{R}_{+} \rightarrow \mathbb{R}_{+}$such that for all $y \geq 0, p^{(H)}(y)=p\left(\frac{y}{H}\right)$, where $p($.$) is the function that was defined and used in$ earlier sections.

Let $u$ denote the common utility function of all the sellers, $c$ the common cost function of all the sellers and $\bar{x}>0$ their common plant capacity.

The Positivity Assumption now reduces to the following:

There exists $\hat{x}, \hat{y} \geq 0$ with $\hat{x}+\hat{y} \leq \bar{x}$, such that $p(\bar{x}) \hat{y}^{\smile} c(\hat{x}+\hat{y})>0$.

A competitive equilibrium strategy $<\left(x^{* g}, y^{* g}\right) \mid g=1, \ldots, H>$ will be said to be symmetric if for all $g, h \in\{1, \ldots, H\}:\left(x^{* g}, y^{* g}\right)=\left(x^{* h}, y^{* h}\right)$.

Let $x^{*}(H)$ denote the quantity of the produced good consumed by each seller at a symmetric competitive equilibrium with $H$ identical sellers and let $y^{*}(H)$ denote the quantity of the produced good sold by each seller at a symmetric competitive equilibrium with $H$ identical sellers.

In what follows we will denote a symmetric competitive equilibrium strategy with $H$ identical sellers by the ordered pair $\left(x^{*}(H), y^{*}(H)\right)$.

A Cournotian equilibrium strategy $<\left(x^{c g}, y^{c g}\right) \mid g=1, \ldots, H>$ will be said to be symmetric if for all $g, h \in\{1, \ldots, H\}:\left(x^{c g}, y^{c g}\right)=\left(x^{c h}, y^{c h}\right)$.

Let $x^{c}(H)$ denote the quantity of the produced good consumed by each seller at a symmetric Cournotian equilibrium with $H$ identical sellers and let $y^{c}(H)$ denote the quantity of the produced good sold by each seller at a symmetric Cournotian equilibrium with $H$ identical sellers.

In what follows we will denote a symmetric Cournotian equilibrium strategy profile with $\mathrm{H}$ identical sellers by the ordered pair $\left(x^{c}(H), y^{c}(H)\right)$.

Corollary of Proposition 1: Suppose twice continuous differentiability of cost functions. Let $\left(x^{*}(H), y^{*}(H)\right)$ denote a symmetric competitive equilibrium strategy. Then either $(a) p\left(y^{*}(H)\right)=p^{(H)}\left(H y^{*}(H)\right)=M c\left(x^{*}(H)+\right.$ 
$\left.y^{*}(H)\right) \operatorname{or}(b) x^{*}(H)+y^{*}(H)=\bar{x}$.

Corollary of Proposition 2: Suppose twice continuous differentiability of cost functions. Let $\left(x^{c}(H), y^{c}(H)\right)$ denote a Cournotian equilibrium strategy. Then either $(a) p\left(y^{c}(H)\right)+\frac{y^{c}(H)}{H} p^{\prime}\left(y^{c}(H)\right)=M c\left(x^{c}(H)+y^{c}(H)\right)$ or $(b) x^{c}(H)+y^{c}(H)=\bar{x}$.

Suppose in addition to what has been assumed before the following condition.

Twice continuous differentiability and strong concavity of utility functions:

(i) $u$ is twice continuously differentiable on $\mathbb{R}_{++}^{2}$ with $D u(\xi, \eta)>0$ and $D^{2} u(\xi, \eta)$ negative definite;

(ii) for all $\eta>0, \lim _{\xi \rightarrow 0} \frac{\partial u(\xi, \eta)}{\partial \xi}=+\infty$ and $\lim _{\xi \rightarrow \infty} \frac{\partial u(\xi, \eta)}{\partial \xi}=0$;

(iii) for all $\xi>0, \lim _{\eta \rightarrow 0} \frac{\partial u(\xi, \eta)}{\partial \eta}=+\infty$ and $\lim _{\eta \rightarrow \infty} \frac{\partial u(\xi, \eta)}{\partial \eta}=0$;

(iv) for all $\xi>0$ and $\eta>0, \frac{\partial^{2} u(\xi, \eta)}{\partial \xi \partial \eta} \geq 0$.

As a consequence of these properties it follows that if $\xi, \eta, \xi^{\prime}, \eta^{\prime} \in \mathbb{R}_{++}$ with $\xi^{\prime} \geq \xi, \eta^{\prime} \leq \eta$ and one of the latter two inequalities being strict then $\frac{D_{2} u\left(\xi^{\prime}, \eta^{\prime}\right)}{D_{1} u\left(\xi^{\prime}, \eta^{\prime}\right)}>\frac{D_{2} u(\xi, \eta)}{D_{1} u(\xi, \eta)}$.

Consider the case of a symmetric competitive equilibrium strategy profile. $\left(x^{*}, y^{*}\right)$ is a symmetric competitive equilibrium strategy in a market with $H$ sellers if and only if $\left(x^{*}, y^{*}\right)$ solves

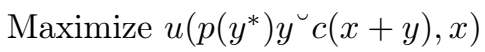

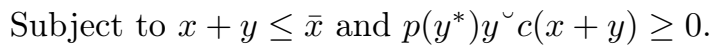

Note: From the above it follows that a symmetric competitive market equilibrium strategy is independent of the number of sellers in the market.

Under twice continuous differentiability of cost functions and twice continuous differentiability of utility functions, $\left(x^{*}, y^{*}\right)$ solves the above problem if and only if there exists $\lambda \geq 0$ such that

(i) $\left(p\left(y^{*}\right)^{\smile} M c\left(x^{*}+y^{*}\right)\right) D_{1} u\left(p\left(y^{*}\right) y^{* \smile c} c\left(x^{*}+y^{*}\right), x^{*}\right)-\lambda \leq 0$ and $\left[\left(p\left(y^{*}\right)^{\smile} M c\left(x^{*}+y^{*}\right)\right) D_{1} u\left(p\left(y^{*}\right) y^{*} c\left(x^{*}+y^{*}\right), x^{*}\right)-\lambda\right] y^{*}=0$.

$(i i)^{\smile} M c\left(x^{*}+y^{*}\right) D_{1} u\left(p\left(y^{*}\right) y^{*} c\left(x^{*}+y^{*}\right), x^{*}\right)+D_{2} u\left(p\left(y^{*}\right) y^{*} c\left(x^{*}+y^{*}\right), x^{*}\right)-$ $\lambda \leq 0$ and $\left[{ }^{\sim} M c\left(x^{*}+y^{*}\right) D_{1} u\left(p\left(y^{*}\right) y^{*} c\left(x^{*}+y^{*}\right), x^{*}\right)+D_{2} u\left(p\left(y^{*}\right) y^{*} c\left(x^{*}+\right.\right.\right.$ $\left.\left.\left.y^{*}\right), x^{*}\right)-\lambda\right] x^{*}=0$.

In the above $D_{i} u($.$) stands for the partial derivative of u$ with respect to the 
$i^{\text {th }}$ coordinate.

We already know by Claim 1 that at a competitive equilibrium $p\left(y^{*}\right) y^{*}>0$.

Thus,

$$
\left(p\left(y^{*}\right)^{\smile} M c\left(x^{*}+y^{*}\right)\right) D_{1} u\left(p\left(y^{*}\right) y^{*} c\left(x^{*}+y^{*}\right), x^{*}\right)-\lambda=0 .
$$

If $p\left(y^{*}\right) y^{*} c\left(x^{*}+y^{*}\right)=0$ and $x^{*}=0$, then $u\left(p\left(y^{*}\right) y^{*} c\left(x^{*}+y^{*}\right), x^{*}\right)=$ $u(0,0)$. However, we have seen several times in the course of proofs in earlier theorems that by the Positivity Assumption $u\left(p\left(y^{*}\right) y^{*} c c\left(x^{*}+y^{*}\right), x^{*}\right)>u(0,0)$.

Thus, $p\left(y^{*}\right) y^{*} c\left(x^{*}+y^{*}\right)=0$ implies $x^{*}>0$.

Hence, from (ii) we get

${ }^{\smile} M c\left(x^{*}+y^{*}\right) D_{1} u\left(p\left(y^{*}\right) y^{*} c\left(x^{*}+y^{*}\right), x^{*}\right)+D_{2} u\left(p\left(y^{*}\right) y^{* \smile} c\left(x^{*}+y^{*}\right), x^{*}\right)-\lambda=0$.

Since $x^{*}>0$ implies $M c\left(x^{*}+y^{*}\right)>0$ and since $\lim _{\eta \rightarrow 0} D_{2} u(\xi, \eta)=+\infty$ if $\eta>0$, the above equality would be violated if $p\left(y^{*}\right) y^{*} c\left(x^{*}+y^{*}\right)=0$ and $x^{*}>0$.

Thus, $p\left(y^{*}\right) y^{* \smile} c\left(x^{*}+y^{*}\right)>0$.

Since $\lim _{\eta \rightarrow 0} D_{2} u(\xi, \eta)=+\infty$ if $\xi>0, x^{*}=0$, would violate condition (ii) above. Thus, $x^{*}>0$ and so ${ }^{\cup} M c\left(x^{*}+y^{*}\right) D_{1} u\left(p\left(y^{*}\right) y^{*} c\left(x^{*}+y^{*}\right), x^{*}\right)+$ $D_{2} u\left(p\left(y^{*}\right) y^{*} c\left(x^{*}+y^{*}\right), x^{*}\right)-\lambda=0$.

We summarize the above discussion in the following proposition.

Proposition 3: Suppose Positivity Assumption, twice continuous differentiability of cost functions and twice continuous differentiability and strong concavity of utility functions. Then $\left(x^{*}, y^{*}\right)$ is a symmetric competitive equilibrium strategy if and only if:

$$
\begin{aligned}
& \text { (i) } x^{*}>0, y^{*}>0 . \\
& (i i) p\left(y^{*}\right) y^{* \smile} c\left(x^{*}+y^{*}\right)>0 . \\
& (\text { iii }) p\left(y^{*}\right) D_{1} u\left(p\left(y^{*}\right) y^{* \smile} c\left(x^{*}+y^{*}\right), x^{*}\right)=D_{2} u\left(p\left(y^{*}\right) y^{* \smile} c\left(x^{*}+y^{*}\right), x^{*}\right) . \\
& (i v)\left(p\left(y^{*}\right)^{\cup} M c\left(x^{*}+y^{*}\right)\right) D_{1} u\left(p\left(y^{*}\right) y^{* \smile} c\left(x^{*}+y^{*}\right), x^{*}\right) \geq 0 .
\end{aligned}
$$

We say that a symmetric competitive equilibrium strategy $\left(x^{*}, y^{*}\right)$ is an interior symmetric competitive equilibrium strategy if $p\left(y^{*}\right)=M c\left(x^{*}+y^{*}\right)$.

We can do a similar analysis for symmetric Cournotian equilibrium strategies. 
$\left(x^{c}(H), y^{c}(H)\right)$ is a symmetric Cournotian equilibrium strategy in a market with $H$ sellers if and only if $\left(x^{c}(H), y^{c}(H)\right)$ solves

Maximize $u\left(p^{H}\left(y+(H-1) y^{c}(H)\right) y^{\iota} c(x+y), x\right)$

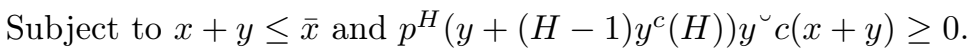

Given our definition of $p H(),.\left(x^{c}(H), y^{c}(H)\right)$ is a symmetric Cournotian equilibrium strategy in a market with $H$ sellers if and only if $\left(x^{c}(H), y^{c}(H)\right)$ solves

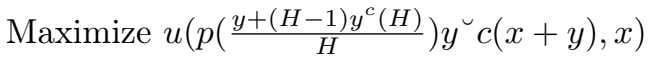

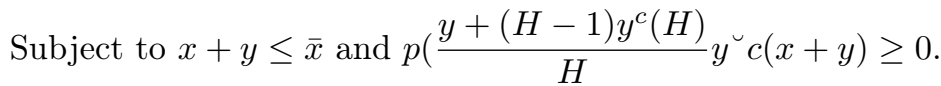

Under twice continuous differentiability of cost functions and twice continuous differentiability and strong concavity of utility functions, $\left(x^{c}(H), y^{c}(H)\right)$ solves the above problem if and only if there exists $\lambda \geq 0$ such that

(i) $\left(p\left(y^{c}(H)\right)+\frac{y^{c}(H)}{H} p^{\prime}\left(y^{c}(H)\right)-M c\left(x^{c}(H)+y^{c}(H)\right)\right) D_{1} u\left(p\left(y^{c}(H)\right) y^{c}(H)-\right.$ $\left.c\left(x^{c}(H)+y^{c}(H)\right), x^{c}(H)\right)-\lambda \leq 0$ and $\left[\left(p\left(y^{c}(H)\right)+\frac{y^{c}(H)}{H} p^{\prime}\left(y^{c}(H)\right)^{\triangleleft} M c\left(x^{c}(H)+\right.\right.\right.$ $\left.\left.\left.y^{c}(H)\right)\right) D_{1} u\left(p\left(y^{c}(H)\right) y^{c}(H)-c\left(x^{c}(H)+y^{c}(H)\right), x^{c}(H)\right)-\lambda\right] y^{c}(H)=0$.

(ii) ${ }^{\triangleleft} M c\left(x^{c}(H)+y^{c}(H)\right) D_{1} u\left(p\left(y^{c}(H)\right) y^{c}(H)^{\smile} c\left(x^{c}(H)+y^{c}(H)\right), y^{c}(H)\right)+$ $D_{2} u\left(p\left(y^{c}(H)\right) y^{c}(H)^{\smile} c\left(x^{c}(H)+y^{c}(H)\right), x^{c}(H)\right)-\lambda \leq 0$ and $\left[{ }^{\triangleleft} M c\left(x^{c}(H)+\right.\right.$ $\left.y^{c}(H)\right) D_{1} u\left(p\left(y^{c}(H)\right) y^{c}(H) \smile c\left(x^{c}(H)+y^{c}(H)\right), x^{c}(H)\right)+D_{2} u\left(p\left(y^{c}(H)\right) y^{c}(H)-\right.$ $\left.\left.c\left(x^{c}(H)+y^{c}(H)\right), x^{c}(H)\right)-\lambda\right] x^{c}(H)=0$.

We already know by Claim 1 that at a competitive equilibrium $p\left(y^{c}(H)\right) y^{c}(H)>$ 0 . Thus,

$$
\begin{aligned}
&\left(p\left(y^{c}(H)\right)+\frac{y^{c}(H)}{H} p^{\prime}\left(y^{c}(H)\right)^{\smile} M c\left(x^{c}(H)+y^{c}(H)\right)\right) . \\
& D_{1} u\left(p\left(y^{c}(H)\right) y^{c}(H)^{\smile} c\left(x^{c}(H)+y^{c}(H)\right), x^{c}(H)\right)-\lambda=0 .
\end{aligned}
$$

If $p\left(y^{c}(H)\right) y^{c}(H)^{\smile} c\left(x^{c}(H)+y^{c}(H)\right)=0$ and $x^{c}(H)=0$, then

$$
u\left(p\left(y^{c}(H)\right) y^{c}(H)^{\smile} c\left(x^{c}(H)+y^{c}(H)\right), x^{c}(H)\right)=u(0,0) .
$$

However, we have seen several times in the course of proofs in earlier theorems that by the Positivity Assumption $u\left(p\left(y^{c}(H)\right) y^{c}(H)^{\smile} c\left(x^{c}(H)+y^{c}(H)\right), x^{c}(H)\right)>$ $u(0,0)$.

Thus, $p\left(y^{c}(H)\right) y^{c}(H)^{\smile} c\left(x^{c}(H)+y^{c}(H)\right)=0$ implies $x^{c}(H)>0$.

Hence, from (ii) we get ${ }^{\triangleleft} M c\left(x^{c}(H)+y^{c}(H)\right) D_{1} u\left(p\left(y^{c}(H)\right) y^{c}(H)^{\smile} c\left(x^{c}(H)+\right.\right.$ $\left.\left.y^{c}(H)\right), x^{c}(H)\right)+D_{2} u\left(p\left(y^{c}(H)\right) y^{c}(H)^{\smile} c\left(x^{c}(H)+y^{c}(H)\right), x^{c}(H)\right)-\lambda=0$. 
Since $x^{c}(H)>0$ and $p\left(y^{c}(H)\right) y^{c}(H)^{\iota} c\left(x^{c}(H)+y^{c}(H)\right)=0$ and since $\lim _{\eta \rightarrow 0} D_{2} u(\xi, \eta)=+\infty$ if $\eta>0$, the above equality would be violated if $p\left(y^{c}(H)\right) y^{c}(H)^{\iota} c\left(x^{c}(H)+y^{c}(H)\right)=0$ and $x^{c}(H)>0$.

Thus, $p\left(y^{c}(H)\right) y^{c}(H)^{\smile} c\left(x^{c}(H)+y^{c}(H)\right)>0$.

Since $\lim _{\eta \rightarrow 0} D_{2} u(\xi, \eta)=+\infty$ if $\xi>0, x^{c}(H)=0$, would violate condition (ii) above. Thus, $x^{c}(H)>0$ and so

$$
\begin{aligned}
& { }^{\hookrightarrow} M c\left(x^{c}(H)+y^{c}(H)\right) . \\
& \quad D_{1} u\left(p\left(y^{c}(H)\right) y^{c}(H)^{\triangleleft} c\left(x^{c}(H)+y^{c}(H)\right), x^{c}(H)\right) \\
& \quad+D_{2} u\left(p\left(y^{c}(H)\right) y^{c}(H)^{\smile} c\left(x^{c}(H)+y^{c}(H)\right), x^{c}(H)\right)-\lambda=0 .
\end{aligned}
$$

We summarize the above discussion in the following proposition.

Proposition 4: Suppose Positivity Assumption, twice continuous differentiability of cost functions and twice continuous differentiability and strong concavity of utility functions. Then $\left(x^{c}(H), y^{c}(H)\right)$ is a symmetric Cournotian equilibrium strategy if and only if:

$$
(i) x^{c}(H)>0, y^{c}(H)>0 .
$$

(ii) $p\left(y^{c}(H)\right) y^{c}(H)^{\smile} c\left(x^{c}(H)+y^{c}(H)\right)>0$.

$(i i i)\left(p\left(y^{c}(H)\right)+\frac{y^{c}(H)}{H} p^{\prime}\left(y^{c}(H)\right)\right) D_{1} u\left(p\left(y^{c}(H)\right) y^{c}(H) \backsim c\left(x^{c}(H)+y^{c}(H)\right)\right.$, $\left.x^{c}(H)\right)=D_{2} u\left(p\left(y^{c}(H)\right) y_{c}(H)^{\smile} c\left(x^{c}(H)+y^{c}(H)\right), x^{c}(H)\right)$.

$$
(i v)\left(p\left(y^{c}(H)\right)+\frac{y^{c}(H)}{H} p^{\prime}\left(y^{c}(H)\right)^{\backsim} M c\left(x^{c}(H)+y^{c}(H)\right)\right) D_{1} u\left(p\left(y^{c}(H)\right) y^{c}(H)\right.
$$
$\left.{ }^{u} c\left(x^{c}(H)+y^{c}(H)\right), x^{c}(H)\right) \geq 0$.

We say that a symmetric Cournotian equilibrium strategy in a market with $\mathrm{H}$ sellers $\left(x^{*}, y^{*}\right)$ is an interior symmetric Cournotian equilibrium strategy if $p\left(y^{c}(H)\right)+\frac{y^{c}(H)}{H} p^{\prime}\left(y^{c}(H)\right)=M c\left(x^{c}(H)+y^{c}(H)\right)$.

In what follows we will assume the following.

Strong Law of Demand: $p^{\prime}<0$ on $\{y>0: p(y)>0\}$.

Lemma 3 Suppose $\left(x^{*}, y^{*}\right)$ and $\left(x^{* *}, y^{* *}\right)$ are two distinct interior symmetric competitive equilibrium strategies. Then the following are satisfied:

(i) $x^{*} \neq x^{* *}, y^{*} \neq y^{* *}$ and $x^{*}+y^{*} \neq x^{* *}+y^{* *}$.

(ii) $y^{*}>y^{* *}$ implies, $x^{* *}>x^{*}, x^{* *}+y^{* *}>x^{*}+y^{*}$ and $p\left(y^{* *}\right) y^{* *}-c\left(x^{* *}+\right.$ $\left.y^{* *}\right)>p\left(y^{*}\right) y^{*}-c\left(x^{*}+y^{*}\right)$. Thus, $u\left(p\left(y^{* *}\right) y^{* *}-c\left(x^{* *}+y^{* *}\right), x^{* *}\right)>u\left(p\left(y^{*}\right) y^{*}-\right.$ $\left.c\left(x^{*}+y^{*}\right), x^{*}\right)$. 
Proof: Suppose $\left(x^{*}, y^{*}\right)$ and $\left(x^{* *}, y^{* *}\right)$ are two distinct interior symmetric competitive equilibrium strategies.

Towards a contradiction suppose $x^{*}+y^{*}=x^{* *}+y^{* *}$. Then $x^{*} \neq x^{* *}, y^{*} \neq$ $y^{* *}$. Without loss of generality suppose $y^{*}>y^{* *}$. Thus $x^{*}<x^{* *}$. Since $M c\left(x^{*}+y^{*}\right)=M c\left(x^{* *}+y^{* *}\right)$, it must be that $p\left(y^{*}\right)=p\left(y^{* *}\right)$. But $y^{*}>y^{* *}$ and the Strong Law of Demand implies $p\left(y^{*}\right)<p\left(y^{* *}\right)$ leading to a contradiction. Thus, $x^{*}+y^{*} \neq x^{* *}+y^{* *}$.

Suppose towards a contradiction $x^{*}=x^{* *}$. Thus $x^{*}+y^{*} \neq x^{* *}+y^{* *}$ implies $y^{*} \neq y^{* *}$. Without loss of generality suppose $y^{*}>y^{* *}$. Thus, $x^{*}+y^{*}>$ $x^{* *}+y^{* *}$.Thus, $M c\left(x^{*}+y^{*}\right) \geq M c\left(x^{* *}+y^{* *}\right)$.

Since $p\left(y^{*}\right)=M c\left(x^{*}+y^{*}\right)$ and $p\left(y^{* *}\right)=M c\left(x^{* *}+y^{* *}\right)$ we get $p\left(y^{*}\right) \geq p\left(y^{* *}\right)$ which since $y^{*}>y^{* *}$ contradicts the Strong Law of Demand. Thus, $x^{*} \neq x^{* *}$.

Now suppose $y^{*}=y^{* *}$. Since $x^{*} \neq x^{* *}$, without loss of generality suppose $x^{*}>x^{* *}$. Thus, $x^{*}+y^{*}>x^{* *}+y^{* *}$. Hence $c\left(x^{*}+y^{*}\right)>c\left(x^{* *}+y^{* *}\right)$. Thus, $p\left(y^{*}\right) y^{*}-c\left(x^{*}+y^{*}\right)<p\left(y^{* *}\right) y^{* *}-c\left(x^{* *}+y^{* *}\right)$.

But $p\left(y^{*}\right) y^{*}-c\left(x^{*}+y^{*}\right)<p\left(y^{* *}\right) y^{* *}-c\left(x^{* *}+y^{* *}\right)$ and $x^{*}>x^{* *}$ implies $p\left(y^{* *}\right)=\frac{D_{2} u\left(\xi^{\prime}, \eta^{\prime}\right)}{D_{1} u\left(\xi^{\prime}, \eta^{\prime}\right)}>\frac{D_{2} u(\xi, \eta)}{D_{1} u(\xi, \eta)}=p(y *)$, where $(\xi, \eta)=\left(p\left(y^{*}\right) y^{*}-c\left(x^{*}+\right.\right.$ $\left.\left.y^{*}\right), x^{*}\right)$ and $\left(\xi^{\prime}, \eta^{\prime}\right)=\left(p\left(y^{* *}\right) y^{* *}-c\left(x^{* *}+y^{* *}\right), x^{* *}\right)$. But this contradicts $y^{*}=y^{* *}$ and proves (i).

To prove (ii) suppose $y^{*}>y^{* *}$. Thus by the Strong Law of Demand, $p\left(y^{*}\right)<$ $p\left(y^{* *}\right)$. Thus, $M c\left(x^{*}+y^{*}\right)<M c\left(x^{* *}+y^{* *}\right)$. Thus, $c\left(x^{*}+y^{*}\right)<c\left(x^{* *}+y^{* *}\right)$ and so $x^{*}+y^{*}<x^{* *}+y^{* *}$ and further $x^{*}<x^{* *}$.

Let $(\xi, \eta)=\left(p\left(y^{*}\right) y^{*}-c\left(x^{*}+y^{*}\right), x^{*}\right) \operatorname{and}\left(\xi^{\prime}, \eta^{\prime}\right)=\left(p\left(y^{* *}\right) y^{* *}-c\left(x^{* *}+\right.\right.$ $\left.\left.y^{* *}\right), x^{* *}\right)$.

$$
\text { If } p\left(y^{*}\right) y^{*}-c\left(x^{*}+y^{*}\right) \geq p\left(y^{* *}\right) y^{* *}-c\left(x^{* *}+y^{* *}\right) \text {, then }
$$

$$
p\left(y^{*}\right)=\frac{D_{2} u(\xi, \eta)}{D_{1} u(\xi, \eta)} \frac{\left(D_{2} u\left(\xi^{\prime}, \eta^{\prime}\right)\right.}{D_{1} u\left(\xi^{\prime}, \eta^{\prime}\right)}=p\left(y^{* *}\right)
$$

which combined with $y^{*}>y^{* *}$ contradicts the Strong Law of Demand. The last part of (ii) follows from the weak monotonicity of $u$. This proves (ii). Q.E.D.

We are now in a position to state and prove the main result of this section.

Theorem 5 Suppose Positivity Assumption, twice continuous differentiability of cost functions, twice continuous differentiability and strong concavity of utility 
functions and the Strong Law of Demand. Let $\left\langle\left(x^{c}(H), y^{c}(H) \mid H \in \mathbb{N}>\right.\right.$ be a sequence of interior symmetric Cournotian equilibrium strategies. Then it has a convergent subsequence and each convergent subsequence converges to an interior symmetric competitive equilibrium strategy. If there is a unique interior symmetric competitive equilibrium strategy then the sequence itself converges to it.

Proof: Since $<\left(x^{c}(H), y^{c}(H) \mid H \in \mathbb{N}>\right.$ lies in the compact set $\{(x, y) \in$ $\left.\mathbb{R}_{+}^{2} \mid x+y \leq \bar{x}\right\}$ it has a convergent subsequence. Hence it has a convergent subsequence converging to a point in $(x, y) \in \mathbb{R}_{+}^{2} \mid x+y \leq \bar{x}$.

Without loss of generality and to save extra notation suppose the sequence itself is convergent and converges to finite vector $\left(x^{*}, y^{*}\right)$. Since $p($.$) is continuous,$ $p^{\prime}($.$) is continuous on \{y>0 \mid p(y)>0\}$ and $\mathrm{p}\left(y^{c}(H)\right)+\frac{y^{c}(H)}{H} p^{\prime}\left(y^{c}(H)\right) \in(0, p(0)]$ for all $H, \lim _{H \rightarrow \infty} p\left(y^{c}(H)\right)=\lim _{H \rightarrow \infty}\left[p\left(y^{c}(H)\right)+\frac{y^{c}(H)}{H} p^{\prime}\left(y^{c}(H)\right)\right] \in[0, p(0)]$. By property (iii) of Proposition 4 and (iv) of the requirements on $u$ (made in this section), it is not possible that $x^{*}>0$ and $y^{*}=0$.

Since $u\left(p\left(y^{c}(H)\right) y^{c}(H)-c\left(x^{c}(H)+y^{c}(H)\right), x^{c}(H)\right) \geq u(p(\bar{x})(\hat{y}-\epsilon)-c(\hat{x}+$ $\hat{y}), \hat{x}+\epsilon)>u(0,0)$, for $\epsilon>0$ sufficiently small such that $p(\bar{x})(\hat{y}-\epsilon)-c(\hat{x}+\hat{y})>0$, it is not possible that $x^{*}=0=y^{*}$.

If $y^{*}>0$ and $x^{*}=0$, then by property (iii) of Proposition 4 and (iv) of the requirements on $\mathrm{u}$ (made in this section), $\lim _{H \rightarrow \infty} p\left(y^{c}(H)\right)=\lim _{H \rightarrow \infty}\left[p\left(y^{c}(H)\right)+\right.$ $\left.\frac{y^{c}(H)}{H} p^{\prime}\left(y^{c}(H)\right)\right]=0$.

However, $p\left(y^{c}(H)\right) \geq p(\bar{x})>0$ for all $H$ implies $\lim _{H \rightarrow \infty} p\left(y^{c}(H)\right)>0$, leading to a contradiction. Thus, $x^{*}>0$ and $y^{*}>0$. This satisfies (i) of Proposition 3.

Now, $p\left(y^{c}(H)\right) y^{c}(H)-c\left(x^{c}(H)+y^{c}(H) \geq p(\bar{x}) \hat{y}-c(\hat{x}+\hat{y})>0\right.$ for all $\mathrm{H}$ implies $p\left(y^{*}\right) y^{*}-c\left(x^{*}+y^{*}\right)=\lim _{H \rightarrow \infty}\left[p\left(y^{c}(H)\right)-c\left(x^{c}(H)+y^{c}(H)\right)\right] \geq$ $p(\bar{x}) \hat{y}-c(\hat{x}+\hat{y})>0$. This satisfies (ii) of Proposition 3 .

Since

$$
\begin{array}{r}
\left(p\left(y^{c}(H)\right)+\frac{y^{c}(H)}{H} p^{\prime}\left(y^{c}(H)\right)\right) D_{1} u\left(p\left(y^{c}(H)\right) y^{c}(H) \smile c\left(x^{c}(H)+y^{c}(H)\right), x^{c}(H)\right)= \\
D_{2} u\left(p\left(y^{c}(H)\right) y^{c}(H)^{\smile} c\left(x^{c}(H)+y^{c}(H)\right), x^{c}(H)\right)
\end{array}
$$

for all $H$, taking limits of both sides and appealing to the continuity of all the functions that are involved in the above equations, we get

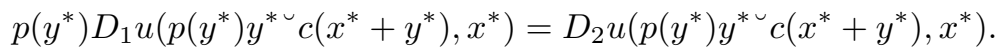

This satisfies (iii) of Proposition 3.

Since

$$
\begin{aligned}
& \left(p\left(y^{c}(H)\right)+\frac{y^{c}(H)}{H} p^{\prime}\left(y^{c}(H)\right)^{\smile} M c\left(x^{c}(H)+\right.\right. \\
& \left.\left.y^{c}(H)\right)\right) D_{1} u\left(p\left(y^{c}(H)\right) y^{c}(H) \smile c\left(x^{c}(H)+y^{c}(H)\right), x^{c}(H)\right) \geq 0
\end{aligned}
$$


for all $H$, taking limits of both sides and appealing to the continuity of all the functions that are involved in the above equations, we get

$$
\left(p\left(y^{*}\right)^{\smile} M c\left(x^{*}+y^{*}\right)\right) D_{1} u\left(p\left(y^{*}\right) y^{* \smile} c\left(x^{*}+y^{*}\right), x^{*}\right) \geq 0 .
$$

This satisfies (iv) of Proposition 3.

Hence by Proposition $3,\left(x^{*}, y^{*}\right)$ is an interior symmetric competitive equilibrium.

Finally suppose $\left(x^{*}, y^{*}\right)$ is the unique interior symmetric competitive equilibrium. Towards a contradiction suppose the given sequence does not converge to $\left(x^{*}, y^{*}\right)$. Then there exists $\delta>0$ such that the (Euclidean) distance of $\left(x^{c}(H), y^{c}(H)\right.$ from $\left(x^{*}, y^{*}\right)$ is greater than or equal to $\delta$ infinitely often. Thus there is a subsequence of the given sequence such that the distance of each term of the subsequence from $\left(x^{*}, y^{*}\right)$ is greater than or equal to $\delta$. Since this subsequence lies in a compact set it has a convergent subsequence which by what we have established above converges to an interior symmetric competitive equilibrium strategy. Since $\left(x^{*}, y^{*}\right)$ is the unique interior symmetric competitive equilibrium this subsequence must converge to $\left(x^{*}, y^{*}\right)$. This contradicts that the distance of every term of the subsequence from $\left(x^{*}, y^{*}\right)$ is greater than or equal to $\delta$. Hence the sequence itself converges to $\left(x^{*}, y^{*}\right)$. Q.E.D.

\section{The Bertrand Edgeworth game and its pure strategy equilibria:}

In this section we assume that all sellers are identical in every respect.

Let $D: \mathbb{R}_{+} \rightarrow \mathbb{R}_{+}$be the demand function corresponding to the inverse demand function $p($.$) , i.e. for all p \in \mathbb{R}_{+}, D(p)=\min \left\{y \in R_{+} \mid p(y)=p\right\}$. In this section(as in the previous one) we assume that $p($.$) satisfies the Strong$ Law of Demand.

Let $s \geq 0$ and $\bar{x}>0$ be constants such that such that the common cost function is given by $c(\xi)=s \xi$ for all $\xi \in[0, \bar{x}]$ and $c(\xi)=+\infty$ for all $\xi>\bar{x}$. Thus $\bar{x}$ is the common production capacity of all the sellers.

As before we assume that the Positivity Assumption is satisfied.

A trivial consequence of the Positivity Assumption (which in this case is $p(H \bar{x})>s)$ is that $H \bar{x}<D(s)$.

Let $u$ be the common utility function of all the sellers and in addition to what we have assumed about $\mathrm{u}$ in section 2 , we assume now that $u$ is strictly 
concave on the set $\left\{(m, \xi) \in \mathbb{R}_{+}^{2} \mid u(m, \xi)>u(0,0)\right\}$.

The Bertrand Edgeworth game is a price setting game, where each seller $h \in\{1, \ldots, H\}$ sets a price $p_{h} \geq s$. In the literature on Bertrand Edgeworth game it is not usually assumed that individual prices should not be lower than unit costs of production. However, setting a price which is less than unit costs is an irrational strategy and we make our assumption in order to save time discussing such theoretically futile possibilities.

A strategy profile denoted $\vec{P}$ is an $H$-tuple $\left(p^{1}, p^{2}, \ldots, p^{H}\right)$ such that $p^{h}$ is the price strategy of seller $h$.

At a strategy profile $\vec{p}$ let $\left\{p^{(1)}, p^{(2)}, \ldots, p^{(K)}\right\}$ denote the set of $K$-distinct prices announced by the sellers,

i.e. for all $h \in\{1, \ldots, H\}, p^{h} \in\left\{p^{(1)}, p^{(2)}, \ldots, p^{(K)}\right\}$ and for all $k \in\{1, \ldots, K\}$, there exists $h \in\{1, \ldots, H\}$ such that $p^{h}=p^{(k)}$. Suppose $p^{(1)}<p^{(2)}<\ldots<$ $p^{(K)}$.

Let $I^{(k)}(\vec{p})=\left\{h \mid p^{h}=p^{(k)}\right\}$ and let $\#\left[I^{(k)}(\vec{p})\right]$ denote its cardinality.

Let $\left(x^{(1)}(\vec{p}), y^{(1)}(\vec{p})\right)$ be a solution to the problem:

Maximize $u\left(p^{(1)} \eta-s(\xi+\eta), \xi\right)$

subject to $\xi+\eta \leq \bar{x}, \eta \leq \frac{D\left(p^{(1)}\right)}{\#\left[I^{(1)}(\vec{p})\right]}, p^{(1)} \eta-s(\xi+\eta) \geq 0$ and $\xi, \eta \geq 0$.

Having defined $\left(x^{(t)}(\vec{p}), y^{(t)}(\vec{p})\right)$ for $t<k$, define $\left(x^{(k)}(\vec{p}), y^{(k)}(\vec{p})\right)=(0,0)$ for all $k \in\{t+1, \ldots, K\}$ if $D\left(p^{(t)}\right)=\sum_{\tau=1}^{t} y^{(\tau)}(\vec{p})\left(\#\left[I^{\tau}(\vec{p})\right]\right)$; if $D\left(p^{(t)}\right)>$ $\sum_{\tau=1}^{t} y^{(\tau)}(\vec{p})\left(\#\left[I^{\tau}(\vec{p})\right]\right)$ then define $\left(x^{(t+1)}(\vec{p}), y^{(t+1)}(\vec{p})\right)$ as a solution to the problem

Maximize $u\left(p^{(t+1)} \eta-s(\xi+\eta), \xi\right)$

subject to $\xi+\eta \leq \bar{x}, \eta \leq \frac{D\left(p^{(t+1)}\right)-\sum_{\tau=1}^{t} y^{(\tau)}(\vec{p})\left(\#\left[I^{(\tau)}(\vec{p})\right]\right.}{\#\left[I^{(t+1)}(\vec{p})\right]}, p^{(t+1)} \eta-s(\xi+\eta) \geq 0$ and $\xi, \eta \geq 0$.

The procedure stops as soon as $\left(x^{(K)}(\vec{p}), y^{(K)}(\vec{p})\right)$ has been defined.

Given a strategy profile $\vec{p}$, a real number $\pi \geq s$ and $h \in\{1, \ldots, H\}$, let $\vec{p}^{-h}$ denote the $(H-1)$-tuple $\left(p^{1}, \ldots, p^{h-1}, p^{h+1}, \ldots, p^{H}\right)$ and $\vec{p}^{-h} \mid \pi$ denote the strategy profile $\left(p^{1}, \ldots, p^{h-1}, \pi, p^{h+1}, \ldots, p^{H}\right)$. 
A strategy profile $\vec{p}$ such that for all $h=1, \ldots, H$ and $\pi \geq s$,

$$
\begin{aligned}
& u\left(p^{h} y^{(k)}(\vec{p})^{\smile} s\left(x^{(k)}(\vec{p})+y^{(k)}(\vec{p})\right), x^{(k)}(\vec{p})\right) \geq \\
& u\left(\pi y^{(j)}\left(\vec{p}^{-h} \mid \pi\right)^{\smile} s\left(x^{(j)}\left(\vec{p}^{-h} \mid \pi\right)+y^{(j)}\left(\vec{p}^{-h} \mid \pi\right)\right), x^{(j)}\left(\vec{p}^{-h} \mid \pi\right)\right),
\end{aligned}
$$

where $h \in I^{(k)}(\vec{p}) \cap I^{(j)}\left(\vec{p}^{-h} \mid \pi\right)$, is said to be a Bertrand Edgeworth (BE) equilibrium.

Lemma 4 Suppose $\vec{p}$ is a BE equilibrium. Then $D\left(p^{(1)}\right)>0$.

Proof: Towards a contradiction suppose, $D\left(p^{(1)}\right)=0$. Then, $D\left(p^{(k)}\right)=0$ for all $k=1, \ldots, K$.

Thus, $y^{(k)}(\vec{p})=0$ for all $k=1, \ldots, K$ and so $x^{(k)}(\vec{p})=0$ for all $k=1, \ldots, K$.

Let $\epsilon>0$ be so chosen that $p^{(1)}-\epsilon>s$ and $D\left(p^{(1)}-\epsilon\right)>0$.

Let $\delta>0$ be such that $\min \left\{D\left(p^{(1)}-\epsilon\right), \bar{x}\right\}>2 \delta>0$.

Then, $\left(p^{(1)}-\epsilon\right) \delta-s \frac{k+1}{k} \delta=\left(p^{(1)}-\epsilon-s\right) \delta-\frac{s}{k} \delta$ for all $k \in \mathbb{N}$, and for $k$ sufficiently large, $\left(p^{(1)}-\epsilon-s\right) \delta-\frac{s}{k} \delta>0$.

Also $x^{(1)}+\frac{\delta}{k}=\frac{\delta}{k}>0$.

Thus, $u\left(\left(p^{(1)}-\epsilon\right)\left(y^{(1)}+\delta\right)-s\left(x^{(1)}+y^{(1)}+\frac{k+1}{k} \delta\right), x^{(1)}+\frac{\delta}{k}\right)=u\left(\left(p^{(1)}-\epsilon\right) \delta-\right.$ $\left.s \frac{k+1}{k} \delta, \frac{\delta}{k}\right)>u(0,0)=u\left(x^{(1)}, y^{(1)}\right)$.

Let $\pi=p^{(1)}-\epsilon$ and let $h \in I^{(1)}(\vec{p})$. Consider the strategy $\vec{p}^{-h} \mid \pi$.

Now the least price at this new strategy is $\pi$ which is chosen by $h$, and all other sellers continue to choose their price strategy as before. Thus, $D\left(p^{g}\right)=0$ for all $g \neq h$ and hence at this new strategy they consume nothing and sell nothing. Hence the utility obtained by a seller $g(\neq h)$ is $u(0,0)$ as before.

However,

$$
\begin{gathered}
u\left(\pi y^{(1)}\left(\vec{p}^{-h} \mid \pi\right)^{\smile} s\left(x^{(1)}\left(\vec{p}^{-h} \mid \pi\right)+y^{(1)}\left(\vec{p}^{-h} \mid \pi\right)\right), x^{(1)}\left(\vec{p}^{-h} \mid \pi\right)\right) \geq \\
u\left(\left(p^{(1)}-\epsilon\right) \delta-s \frac{k+1}{k} \delta, \frac{\delta}{k}\right)>u(0,0)= \\
u\left(p^{h} y^{(1)}(\vec{p})^{\smile} s\left(x^{(1)}(\vec{p})+y^{(1)}(\vec{p})\right), x^{(1)}(\vec{p})\right)
\end{gathered}
$$

and $h \in I^{(1)}(\vec{p}) \cap I^{(1)}\left(\vec{p}^{-h} \mid \pi\right)$, for $k \in \mathbb{N}$ sufficiently large, contradicting $\vec{p}$ is a $\mathrm{BE}$ equilibrium.

This proves the lemma. Q.E.D. 
Lemma 5 Suppose $\vec{p}$ is a BE equilibrium. Then $p^{(1)}>s$.

Proof: Towards a contradiction suppose $p^{(1)}=s$. In this case it is easy to see that $x^{(1)}=0$.

By Positivity Assumption $H \bar{x}<D(s)$. Hence \#[$\left.I^{(1)}(\vec{p})\right] \bar{x}<D(s)$. Let $h \in I^{(1)}(\vec{p})$.

Case 1: $x^{(1)}+y^{(1)}=y^{(1)}<\bar{x}$.

In this case consider $\epsilon>0$ sufficiently small such that $p^{(1)}+\epsilon<p^{(2)}$ and $D\left(p^{(1)}+\epsilon\right)-\left(\# I^{(1)}(\vec{p})^{\sim} 1\right) y^{(1)}>\bar{x}$.

Let $\delta>0$ be such that $x^{(1)}+y^{(1)}+2 \delta<\bar{x}$. Thus, $x^{(1)}+y^{(1)}+\frac{k+1}{k} \delta<\bar{x}$ for all $k \in \mathbb{N}$.

Clearly, for all $k \in \mathbb{N}, y^{(1)}+\frac{k+1}{k} \delta \leq y^{(1)}+2 \delta \leq x^{(1)}+y^{(1)}<\bar{x}<$ $D\left(p^{(1)}+\epsilon\right)-\#\left[I^{(1)}(\vec{p})-1\right] y^{(1)}$.

Further, $\left[\left(p^{(1)}+\epsilon\right)\left(y^{(1)}+\delta\right)^{\triangleleft} s\left(x^{(1)}+y^{(1)}+\frac{k+1}{k} \delta\right)\right]^{\triangleleft}\left[p^{(1)} y^{(1)} \backsim s\left(x^{(1)}+y^{(1)}\right)\right]=$ $(s+\epsilon) \delta+\epsilon y^{(1)} \cup \frac{k+1}{k} \delta s=\epsilon\left(y^{(1)}+\delta\right)-\frac{\delta}{k} s=\epsilon y^{(1)}+\delta\left(\epsilon-\frac{s}{k}\right)>0$ for $k \in \mathbb{N}$ sufficiently large.

Also, $x^{(1)}+\frac{\delta}{k}>x^{(1)}$ and so, $u\left(\left(p^{(1)}+\epsilon\right)\left(y^{(1)}+\delta\right)^{\smile} s\left(x^{(1)}+y^{(1)}+2 \delta\right), x^{(1)}+\delta\right)>$ $u\left(p^{(1)} y^{(1)} \triangleleft s\left(x^{(1)}+y^{(1)}\right), x^{(1)}\right)$ for $k \in \mathbb{N}$ sufficiently large.

Let $\pi=p^{(1)}+\epsilon$ and consider the strategy $\vec{p}^{-h} \mid \pi$.

If $I^{(1)}(\vec{p}) \backslash\{h\}=\phi$, then $h \in I^{(1)}(\vec{p}) \cap I^{(1)}\left(\vec{p}^{-h} \mid \pi\right)$ and

$$
\begin{gathered}
u\left(\pi y^{(1)}\left(\vec{p}^{-h} \mid \pi\right)^{\triangleleft} s\left(x^{(1)}\left(\vec{p}^{-h} \mid \pi\right)+y^{(1)}\left(\vec{p}^{-h} \mid \pi\right), x^{(1)}\left(\vec{p}^{-h} \mid \pi\right)\right) \geq\right. \\
u\left(\epsilon y^{(1)}+\delta\left(\epsilon-\frac{s}{k}\right), \frac{\delta}{k}\right)> \\
u\left(p^{h} y^{(1)}(\vec{p})^{\smile} s\left(x^{(1)}(\vec{p})+y^{(1)}(\vec{p})\right), x^{(1)}(\vec{p})\right),
\end{gathered}
$$

for $k \in \mathbb{N}$ sufficiently large, contradicting $\vec{p}$ is a BE equilibrium.

If $I^{(1)}(\vec{p}) \backslash\{h\} \neq \phi$, then our deviating agent $h \in I^{(1)}(\vec{p}) \cap I^{(2)}\left(\vec{p}^{-h} \mid \pi\right)$.

The problem faced by a seller $g \in I^{(1)}\left(\vec{p}^{-h} \mid \pi\right)=I^{(1)}(\vec{p}) \backslash\{h\}$, is the following:

Maximize $u\left(p^{(1)} \eta-s(\xi+\eta), \xi\right)$

subject to $\xi+\eta \leq \bar{x}, \eta \leq \frac{D\left(p^{(1)}\right)}{\#\left[I^{(1)}(\vec{p})\right]-1}, p^{(1)} \eta-s(\xi+\eta) \geq 0$ and $\xi, \eta \geq 0$. 
Since $y^{(1)}<\frac{D\left(p^{(1)}\right)}{\#\left[I^{(1)}(\vec{p})\right]}<\frac{D\left(p^{(1)}\right)}{\#\left[I^{(1)}(\vec{p})\right]-1},\left(x^{(1)}, y^{(1)}\right)$ is feasible for this problem and from the concavity of $u$ it follows that $\left(x^{(1)}, y^{(1)}\right)$ is optimal for this problem (if not then there would exist a feasible solution for this problem giving higher utility and a convex combination of this new feasible solution and $\left(x^{(1)}, y^{(1)}\right)$, sufficiently close to $\left(x^{(1)}, y^{(1)}\right)$ would be feasible for the earlier problem, and would give a higher utility than what $\left(x^{(1)}, y^{(1)}\right)$ gives, contradicting the definition of $\left.\left(x^{(1)}, y^{(1)}\right)\right)$.

Thus, $u\left(\pi y^{(2)}\left(\vec{p}^{-h} \mid \pi\right)^{\smile} s\left(x^{(2)}\left(\vec{p}^{-h} \mid \pi\right)+y^{(2)}\left(\vec{p}^{-h} \mid \pi\right), x^{(2)}\left(\vec{p}^{-h} \mid \pi\right)\right) \geq u\left(\epsilon y^{(1)}+\right.\right.$ $\left.\delta\left(\epsilon-\frac{s}{k}\right), \frac{\delta}{k}\right)>u\left(p^{h} y^{(1)}(\vec{p}) \backsim s\left(x^{(1)}(\vec{p})+y^{(1)}(\vec{p})\right), x^{(1)}(\vec{p})\right)$.

This contradicts that $\vec{p}$ is a BE equilibrium.

Case 2: $x^{(1)}+y^{(1)}=y^{(1)}=\bar{x}$.

In this case it is easy to see that $y^{(1)}=\bar{x}$ and $x^{(1)}=0$.

Consider $\epsilon>0$ sufficiently small such that $p^{(1)}+\epsilon<p^{(2)}$ and $D\left(p^{(1)}+\epsilon\right)-$ $\left(\# I^{(1)}(\vec{p})^{\cup} 1\right) y^{(1)} \leq \bar{x}$.

Now, $\left[\left(p^{(1)}+\epsilon\right)\left(y^{(1)}-\delta\right)^{\smile} s\left(x^{(1)}+y^{(1)}\right)\right]^{\triangleleft}\left[p^{(1)} y^{(1)}{ }^{\prime} s\left(x^{(1)}+y^{(1)}\right)\right]=-\left(p^{(1)}+\right.$ $\epsilon) \delta+\epsilon y^{(1)}>0$ for $\delta>0$ sufficiently small.

Also, $x^{(1)}+\delta>x^{(1)}$ and so, $u\left(\left(p^{(1)}+\epsilon\right)\left(y^{(1)}-\delta\right)^{\smile} s\left(x^{(1)}+y^{(1)}\right), x^{(1)}+\delta\right)>$ $u\left(p^{(1)} y^{(1)} \backsim s\left(x^{(1)}+y^{(1)}\right), x^{(1)}\right)$.

Let $\pi=p^{(1)}+\epsilon$ and consider the strategy $\vec{p}^{-h} \mid \pi$.

If $I^{(1)}(\vec{p}) \backslash\{h\}=\phi$, then $h \in I^{(1)}(\vec{p}) \cap I^{(1)}\left(\vec{p}^{-h} \mid \pi\right)$ and

$$
\begin{aligned}
u\left(\pi y^{(1)}\left(\vec{p}^{-h} \mid \pi\right)^{\smile} s\left(x^{(1)}\left(\vec{p}^{-h} \mid \pi\right)+y^{(1)}\left(\vec{p}^{-h} \mid \pi\right), x^{(1)}\left(\vec{p}^{-h} \mid \pi\right)\right) \geq\right. & \\
u\left(\epsilon y^{(1)}-\right. & \left.\left(p^{(1)}+\epsilon\right) \delta, \delta\right)> \\
& u\left(p^{h} y^{(1)}(\vec{p})^{\smile} s\left(x^{(1)}(\vec{p})+y^{(1)}(\vec{p})\right), x^{(1)}(\vec{p})\right)
\end{aligned}
$$

for $\delta>0$ sufficiently small.

If $I^{(1)}(\vec{p}) \backslash\{h\} \neq \phi$, then our deviating agent $h \in I^{(1)}(\vec{p}) \cap I^{(2)}\left(\vec{p}^{-h} \mid \pi\right)$.

The problem faced by a seller $g \in I^{(1)}\left(\vec{p}^{-h} \mid \pi\right)=I^{(1)}(\vec{p}) \backslash\{h\}$, is the following:

Maximize $u\left(p^{(1)} \eta-s(\xi+\eta), \xi\right)$

subject to $\xi+\eta \leq \bar{x}, \eta \leq \frac{D\left(p^{(1)}\right)}{\#\left[I^{(1)}(\vec{p})\right]-1}, p^{(1)} \eta-s(\xi+\eta) \geq 0$ and $\xi, \eta \geq 0$.

Since $y^{(1)}<\frac{D\left(p^{(1)}\right)}{\#\left[I^{(1)}(\vec{p})\right]}<\frac{D\left(p^{(1)}\right)}{\#\left[I^{(1)}(\vec{p})\right]-1},\left(x^{(1)}, y^{(1)}\right)$ is feasible for this problem and from the concavity of $u$ it follows that $\left(x^{(1)}, y^{(1)}\right)$ is optimal for this problem (if not then there would exist a feasible solution for this problem giving higher 
utility and a convex combination of this new feasible solution and $\left(x^{(1)}, y^{(1)}\right)$, sufficiently close to $\left(x^{(1)}, y^{(1)}\right)$ would be feasible for the earlier problem, and would give a higher utility than what $\left(x^{(1)}, y^{(1)}\right)$ gives, contradicting the definition of $\left.\left(x^{(1)}, y^{(1)}\right)\right)$.

Thus, $u\left(\pi y^{(2)}\left(\vec{p}^{-h} \mid \pi\right)^{\triangleleft} s\left(x^{(2)}\left(\vec{p}^{-h} \mid \pi\right)+y^{(2)}\left(\vec{p}^{-h} \mid \pi\right), x^{(2)}\left(\vec{p}^{-h} \mid \pi\right)\right) \geq u\left(\epsilon y^{(1)}-\right.\right.$ $\left.\left(p^{(1)}+\epsilon\right) \delta, \delta\right)>u\left(p^{h} y^{(1)}(\vec{p})\left\llcorner s\left(x^{(1)}(\vec{p})+y^{(1)}(\vec{p})\right), x^{(1)}(\vec{p})\right)\right.$ for $\delta>0$ sufficiently small.

This once again contradicts that $\vec{p}$ is a BE equilibrium and proves the lemma. Q.E.D.

An immediate consequence of Lemma 5 is the following.

Lemma 6 Suppose $\vec{p}$ is a BE equilibrium with $\left\{p^{(1)}, \ldots, p^{(K)}\right\}$ being the set of distinct prices quoted at $\vec{p}$ and. Suppose $K>1$. Then, $y^{(1)}(\vec{p})<\frac{D\left(p^{1}\right)}{\# I^{(1)}(\vec{p})}$.

Proof: Towards a contradiction suppose $y^{(1)}(\vec{p})=\frac{D\left(p^{1}\right)}{\# I^{(1)}(\vec{p})}$. Then

$$
\left(x^{(k)}(\vec{p}), y^{(k)}(\vec{p})\right)=(0,0)
$$

$<++>$ for $k=2, \ldots, K$.

By Lemma $5, p^{(1)}>s$. Hence there exists $\epsilon>0$ sufficiently small, such that $p^{(1)}-\epsilon>s$.

By Lemma $2, D\left(p^{(1)}\right)>0$. Thus by Strong Law of Demand $D\left(p^{(1)}-\epsilon\right)>0$.

Let $\pi=p^{(1)}-\epsilon, h \in I^{(2)}(\vec{p})$ and consider, $\vec{p}^{-h} \mid \pi$.

Let $\delta>0$ be such that $2 \delta<\min \left\{\bar{x}, D\left(p^{(1)}-\epsilon\right)\right\}$.

Now $\left(p^{(1)}-\epsilon\right) \delta-s\left(\frac{k+1}{k} \delta\right)=\left(p^{(1)}-\epsilon\right) \delta-\frac{s}{k} \delta>0$ for $k \in \mathbb{N}$ sufficiently large.

Hence $\left(\left(p^{(1)}-\epsilon\right) \delta-s\left(\frac{k+1}{k} \delta\right), \frac{\delta}{k}\right) \in \mathbb{R}_{++}^{2}$ and so $u\left(\left(p^{(1)}-\epsilon\right) \delta-s\left(\frac{k+1}{k} \delta\right), \frac{\delta}{k}\right)>$ $u(0,0)=u\left(p^{h} y^{(2)}(\vec{p})^{\smile} s\left(x^{(2)}(\vec{p})+y^{(2)}(\vec{p})\right), x^{(2)}(\vec{p})\right)$ for $k \in \mathbb{N}$ sufficiently large.

Observe that $\{h\}=I^{(1)}\left(\vec{p}^{-h} \mid \pi\right)$.

Thus, $u\left(\pi y^{(1)}\left(\vec{p}^{-h} \mid \pi\right)^{\smile} s\left(x^{(1)}\left(\vec{p}^{-h} \mid \pi\right)+y^{(1)}\left(\vec{p}^{-h} \mid \pi\right), x^{(1)}\left(\vec{p}^{-h} \mid \pi\right)\right) \geq u\left(\left(p^{(1)}-\right.\right.\right.$ $\left.\epsilon) \delta-s\left(\frac{k+1}{k} \delta\right), \frac{\delta}{k}\right)>u\left(p^{h} y^{(2)}(\vec{p}) \backsim s\left(x^{(2)}(\vec{p})+y^{(2)}(\vec{p})\right), x^{(2)}(\vec{p})\right)$ for $k \in \mathbb{N}$ sufficiently large.

This contradicts that $\vec{p}$ is a $\mathrm{BE}$ equilibrium and proves the lemma. Q.E.D.

The next lemma states if $\vec{p}$ is a BE equilibrium then $p^{h}=p^{(1)}>s$ for all $h=1, \ldots, H$.

Lemma 7 Suppose $\vec{p}$ is a BE equilibrium. Then $p^{h}=p^{(1)}$ for all $h=1, \ldots, H$.

Proof: Towards a contradiction suppose $K>1$ where the set of distinct prices at $\vec{p}$ is $\left\{p^{(1)}, p^{(2)}, \ldots, p^{(K)}\right\}$.

By Lemma 6 , \#[I(1) $(\vec{p})] y^{(1)}(\vec{p})<D\left(p^{(1)}\right)$. 
By Lemma $5, p^{(1)}>s$.

Let $h \in I^{(1)}(\vec{p})$.

Case $1: y^{(1)}(\vec{p})=0$.

Thus, $x^{(1)}(\vec{p})=0$ and so $x^{(1)}(\vec{p})+y^{(1)}(\vec{p})=0<\bar{x}$.

The problem faced by a seller $g \in I^{(1)}(\vec{p})$, is the following:

Maximize $u\left(p^{(1)} \eta-s(\xi+\eta), \xi\right)$

subject to $\xi+\eta \leq \bar{x}, \eta \leq \frac{D\left(p^{(1)}\right)}{\# I^{(1)}(\vec{p})}, p^{(1)} \eta-s(\xi+\eta) \geq 0$ and $\xi, \eta \geq 0$.

By Lemma $4, \frac{D\left(p^{(1)}\right)}{\#\left[I^{(1)}(\vec{p})\right]}>0$ and by Lemma $5, p^{(1)}>s$.

Let $\delta>0$ be such that $2 \delta<\min \left\{\bar{x}, \frac{D\left(p^{(1)}\right)}{\# I^{(1)}(\vec{p})}\right\}$.

Now, $p^{(1)} \delta^{\bullet} \frac{k+1}{k} \delta s=\left(p^{(1)}-s\right) \delta-\frac{s}{k} \delta>0$ for $k \in \mathbb{N}$ sufficiently large.

Also, $x^{(1)}+\frac{\delta}{k}>x^{(1)}$ and so, $u\left(p^{(1)}\left(y^{(1)}+\delta\right)-\frac{k+1}{k} \delta s, x^{(1)}+\frac{\delta}{k}\right)>u(0,0)=$ $u\left(p^{(1)} y^{(1)}-s\left(x^{(1)}+y^{(1)}\right), x^{(1)}\right)$ for $k \in \mathbb{N}$ sufficiently large, contradicting the definition of $\left(x^{(1)}, y^{(1)}\right)$.

Thus, $y^{(1)}(\vec{p})=0$ is not possible.

Case 2: $y^{(1)}(\vec{p})>0$.

Consider $\epsilon>0$ sufficiently small such that $p^{(1)}+\epsilon<p^{(2)}$ and $D\left(p^{(1)}+\epsilon\right)>$ $\#\left[I^{(1)}(\vec{p})\right] y^{(1)}(\vec{p})$.

Now, $\left[\left(p^{(1)}+\epsilon\right)\left(y^{(1)}-\delta\right)^{\triangleleft} s\left(x^{(1)}+y^{(1)}\right)\right]^{\triangleleft}\left[p^{(1)} y^{(1)} \triangleleft s\left(x^{(1)}+y^{(1)}\right)\right]=-\left(p^{(1)}+\right.$ $\epsilon) \delta+\epsilon y^{(1)}>0$ for $\delta>0$ sufficiently small.

Also, $x^{(1)}+\delta>x^{(1)}$ and so, $u\left(\left(p^{(1)}+\epsilon\right)\left(y^{(1)}-\delta\right)^{\iota} s\left(x^{(1)}+y^{(1)}\right), x^{(1)}+\delta\right)>$ $u\left(p^{(1)} y^{(1)} \backsim s\left(x^{(1)}+y^{(1)}\right), x^{(1)}\right)$ for $\delta>0$ sufficiently small.

Let $\pi=p^{(1)}+\epsilon$ and consider $\vec{p}^{-h} \mid \pi$.

If $I^{(1)}\left(\vec{p} \backslash\{h\}=\phi\right.$, then $h \in I^{(1)}(\vec{p}) \cap I^{(1)}\left(\vec{p}^{-h} \mid \pi\right)$ and

$$
\begin{gathered}
u\left(\pi y^{(1)}\left(\vec{p}^{-h} \mid \pi\right)^{\smile} s\left(x^{(1)}\left(\vec{p}^{-h} \mid \pi\right)+y^{(1)}\left(\vec{p}^{-h} \mid \pi\right), x^{(1)}\left(\vec{p}^{-h} \mid \pi\right)\right) \geq\right. \\
u\left(\left(p^{(1)}+\epsilon\right)\left(y^{(1)}-\delta\right)^{\triangleleft} s\left(x^{(1)}+y^{(1)}\right), x^{(1)}+\delta\right)> \\
u\left(p^{h} y^{(1)}(\vec{p})^{\smile} s\left(x^{(1)}(\vec{p})+y^{(1)}(\vec{p})\right), x^{(1)}(\vec{p})\right)
\end{gathered}
$$


for $\delta>0$ sufficiently small.

If $I^{(1)}(\vec{p}) \backslash\{h\} \neq \phi$, then our deviating agent $h \in I^{(1)}(\vec{p}) \cap I^{(2)}\left(\vec{p}^{-h} \mid \pi\right)$.

The problem faced by a seller $g \in I^{(1)}\left(\vec{p}^{-h} \mid \pi\right)=I^{(1)}(\vec{p}) \backslash\{h\}$, is the following:

Maximize $u\left(p^{(1)} \eta-s(\xi+\eta), \xi\right)$

subject to $\xi+\eta \leq \bar{x}, \eta \leq \frac{D\left(p^{(1)}\right)}{\#\left[I^{(1)}(\vec{p})\right]-1}, p^{(1)} \eta-s(\xi+\eta) \geq 0$ and $\xi, \eta \geq 0$.

Since $y^{(1)}<\frac{D\left(p^{(1)}\right)}{\#\left[I^{(1)}(\vec{p})\right]}<\frac{D\left(p^{(1)}\right)}{\#\left[I^{(1)}(\vec{p})\right]-1}\left(x^{(1)}, y^{(1)}\right)$ is feasible for this problem and from the concavity of $u$ it follows that $\left(x^{(1)}, y^{(1)}\right)$ is optimal for this problem (if not then there would exist a feasible solution for this problem giving higher utility and a convex combination of this new feasible solution and $\left(x^{(1)}, y^{(1)}\right)$, sufficiently close to $\left(x^{(1)}, y^{(1)}\right)$ would be feasible for the earlier problem, and would give a higher utility than what $\left(x^{(1)}, y^{(1)}\right)$ gives, contradicting the definition of $\left.\left(x^{(1)}, y^{(1)}\right)\right)$.

Thus, $u\left(\pi y^{(2)}\left(\vec{p}^{-h} \mid \pi\right)^{\smile} s\left(x^{(2)}\left(\vec{p}^{-h} \mid \pi\right)+y^{(2)}\left(\vec{p}^{-h} \mid \pi\right), x^{(2)}\left(\vec{p}^{-h} \mid \pi\right)\right) \geq u\left(\left(p^{(1)}+\right.\right.\right.$ $\left.\epsilon)\left(y^{(1)}-\delta\right)^{\llcorner} s\left(x^{(1)}+y^{(1)}\right), x^{(1)}+\delta\right)>u\left(p^{h} y^{(1)}(\vec{p})^{\iota} s\left(x^{(1)}(\vec{p})+y^{(1)}(\vec{p})\right), x^{(1)}(\vec{p})\right)$ for $\delta>0$ sufficiently small.

This contradicts that $\vec{p}$ is a BE equilibrium and proves the lemma. Q.E.D.

An immediate consequence of lemma 7 is that a $\mathrm{BE}$ equilibrium price is greater than or equal to $p(H \bar{x})$, since every one quotes the same price at a $\mathrm{BE}$ and the aggregate quantity supplied cannot exceed $H \bar{x}$.

Lemma 8 Suppose $\vec{p}$ is a BE equilibrium with $p^{h}=p$ for all $h=1, \ldots, H$. Then each seller sells $\frac{D(p)}{H}$.

Proof: Let $(x, y)$ be the unique solution to the problem

Maximize $u(p \eta-s(\xi+\eta), \xi)$

subject to $\xi+\eta \leq \bar{x}, \eta \leq \frac{D(p)}{H}, p \eta-s(\xi+\eta) \geq 0$ and $\xi, \eta \geq 0$.

Towards a contradiction suppose $y<\frac{D(p)}{H}$.

Consider seller 1 and let $\epsilon>0$ be so small such that $D(p+\epsilon)>H y$.

Let $\delta>0$ be such that $y+\delta<\frac{D(p+\epsilon)}{H}$. 
Then, $(p+\epsilon)(y+\delta)^{\iota} s\left(x+y+\frac{k+1}{k} \delta\right)=p y \leftrightharpoons s(x+y)+\epsilon(y+\delta)+(p-s) \delta-\frac{s}{k} \delta>$ $p y \leftrightharpoons s(x+y)$ for $k \in \mathbb{N}$ sufficiently large.

Also, $x+\frac{\delta}{k}>x$ and so $u\left(\pi(y+\delta)^{\smile} s\left(x+y+\frac{k+1}{k} \delta\right), x+\frac{\delta}{k}\right)>u(p y \leftrightharpoons s(x+y), x)$ for $k \in \mathbb{N}$ sufficiently large.

Let $\pi=p+\epsilon$ and consider the strategy profile $\vec{p}^{-1} \mid \pi .1 \in I^{(2)}\left(\vec{p}^{-1} \mid \pi\right)$.

At this strategy profile the problem faced by a seller $h(\neq 1)$ is given by

Maximize $u(p \eta-s(\xi+\eta), \xi)$

subject to $\xi+\eta \leq \bar{x}, \eta \leq \frac{D(p)}{H-1}, p \eta-s(\xi+\eta) \geq 0$ and $\xi, \eta \geq 0$.

Clearly $y<\frac{D(p)}{H}<\frac{D(p)}{H-1}$ and $(x, y)$ is feasible for this problem. From the concavity of $\mathrm{u}$ it follows that $(x, y)$ is optimal for this problem (if not then there would exist a feasible solution for this problem giving higher utility and a convex combination of this new feasible solution and $(x, y)$, sufficiently close to $(x, y)$ would be feasible for the earlier problem, and would give a higher utility than what $(x, y)$ gives, contradicting the definition of $(x, y))$.

Thus, $u\left(\pi y^{(2)}\left(\vec{p}^{-1} \mid \pi\right)^{\smile} s\left(x^{(2)}\left(\vec{p}^{-1} \mid \pi\right)+y^{(2)}\left(\vec{p}^{-1} \mid \pi\right), x^{(2)}\left(\vec{p}^{-1} \mid \pi\right)\right) \geq u(\pi(y+\right.$ $\left.\delta)^{\smile} s\left(x+y+\frac{k+1}{k} \delta\right), x+\delta\right)>u(p y \triangleleft s(x+y), x)$ for $k \in \mathbb{N}$ sufficiently large.

This contradicts that $\vec{p}$ is BE equilibrium and proves the lemma. Q.E.D.

Our next lemma states that at a BE equilibrium all sellers produce $\bar{x}$ units of the produced good.

LEMma 9 Let $x$ be the amount of the produced good that each seller consumes and $y$ be the amount of the produced good that each seller sells at a BE. Then $x+y=\bar{x}$.

Proof: Towards a contradiction suppose $x+y<\bar{x}$.

Suppose $y=0$. Then $D(p)=H y=0$, contradicting lemma 4. Thus $y>0$.

Consider $\epsilon>0$ sufficiently small such that $p-\epsilon>s$. This is possible, since by lemma $5, p>s$. Observe that $D(p-\epsilon)>D(p)$ so that for $\delta>0$ sufficiently small $x+y+2 \delta<\bar{x}$ and $y+\delta=\frac{D(p)}{H}+\delta<D(p)<D(p-\epsilon)$, since $H>1$.

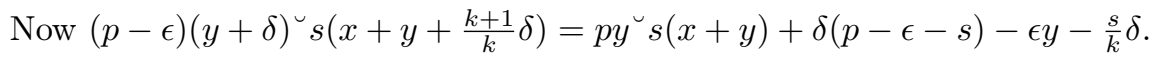

Holding $\delta$ fixed for $\epsilon>0$ sufficiently small and $k \in \mathbb{N}$ sufficiently large,

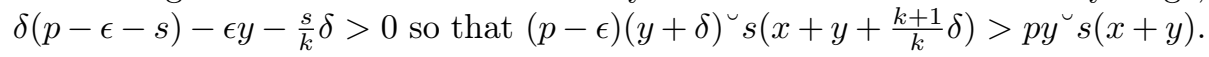


Also $x+\frac{\delta}{k}>x$.

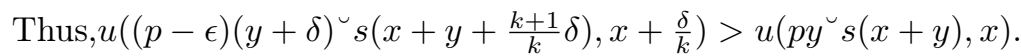

Let $\pi=p-\epsilon$ and consider $\vec{p}^{-1} \mid \pi \cdot\{1\}=I^{(2)}\left(\vec{p}^{-1} \mid \pi\right)$.

Clearly, $u\left(\pi y^{(1)}\left(\vec{p}^{-1} \mid \pi\right)^{\smile} s\left(x^{(1)} \vec{p}^{-1} \mid \pi\right)+y^{(1)}\left(\vec{p}^{-1} \mid \pi\right), x^{(1)}\left(\vec{p}^{-1} \mid \pi\right) \geq u((p-\epsilon)(y+\right.$

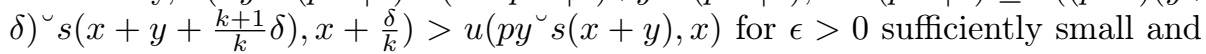
$k \in \mathbb{N}$ sufficiently large.

This contradicts that $\vec{p}$ is BE equilibrium and proves the lemma. Q.E.D.

The next lemma says that in our model, a BE equilibrium is of necessity a competitive equilibrium.

Lemma 10 Let $\vec{p}$ be a BE equilibrium strategy profile with $\vec{p}=(p, p, \ldots, p)$. Then $p$ is a competitive equilibrium price.

Proof: Suppose $\vec{p}$ is BE equilibrium. Then by lemmas 5 and 7 , there exists $p>s$ such that $\vec{p}=(p, \ldots, p)$, i.e. every seller chooses $p$ as his strategy. By lemma $4, D(p)>0$ and by lemma 8 , each seller sells $\frac{D(p)}{H}$. By lemma 9 , if $x$ is the amount of the produced good that each seller consumes and $y$ is the amount of the produced good that each seller sells at $\vec{p}$ then $x+y=\bar{x}$.

We will show that $(x, y)$ is a competitive equilibrium strategy.

Clearly, $p y-s(x+y) \geq 0$.

Note that $(x, y)$ is the unique solution to the following maximization problem:

Maximize $u(p \eta-s(\xi+\eta), \xi)$

subject to $\xi+\eta \leq \bar{x}, \eta \leq \frac{D(p)}{H}, p \eta-s(\xi+\eta) \geq 0$ and $\xi, \eta \geq 0$.

Suppose towards a contradiction $(x, y)$ does not solve

Maximize $u(p \eta-s(\xi+\eta), \xi)$

subject to $\xi+\eta \leq \bar{x}, p \eta-s(\xi+\eta) \geq 0$ and $\xi, \eta \geq 0$.

Then there exists $x^{\prime}, y^{\prime} \geq 0$ with $x^{\prime}+y^{\prime} \leq \bar{x}$ and $\left.p y^{\prime}\right\lrcorner s\left(x^{\prime}+y^{\prime}\right) \geq 0$ such that $u\left(p y^{\prime} \triangleleft s\left(x^{\prime}+y^{\prime}\right), x^{\prime}\right)>u(p y-s(x+y), x)$. 
Since $(x, y)$ solves

Maximize $u(p \eta-s(\xi+\eta), \xi)$

subject to $\xi+\eta \leq \bar{x}, \eta \leq \frac{D(p)}{H}, p \eta-s(\xi+\eta) \geq 0$ and $\xi, \eta \geq 0$.

it is not possible that $y^{\prime} \leq \frac{D(p)}{H}$. Thus, $y^{\prime}>\frac{D(p)}{H}$.

Let $(x(t), y(t))=(x, y)+t\left[\left(x^{\prime}, y^{\prime}\right)^{\smile}(x, y)\right]$ for $0 \leq t \leq 1$.

For all $t \in[0,1],(x(t), y(t))$ satisfies all the constraints of the maximization problem

Maximize $u(p \eta-s(\xi+\eta), \xi)$

subject to $\xi+\eta \leq \bar{x}, p \eta-s(\xi+\eta) \geq 0$ and $\xi, \eta \geq 0$.

Further by the (strict) concavity of $u, u(p y(t)-s(x(t)+y(t)), x(t))>u(p y-$ $s(x+y), x)$ for all $t \in(0,1]$.

Let $\epsilon>0$ be sufficiently so that $p-\epsilon>s$ and $u((p-\epsilon) y(t)-s(x(t)+$ $y(t)), x(t))>u(p y-s(x+y), x)$ for all $t>0$ sufficiently small with $y(t)<$ $D(p-\epsilon)$. This is possible since $y=\frac{D(p)}{H}<D(p)<D(p-\epsilon)$ the last inequality following from the Strong Law of Demand.

Let $\pi=p-\epsilon$ and consider $\vec{p}^{-1} \mid \pi$, i.e. seller 1 deviates unilaterally and chooses the price strategy $\pi$.

Clearly, $I^{(1)}\left(\vec{p}^{-1} \mid \pi\right)=\{1\}$ and

$$
\begin{aligned}
u\left(\pi y^{(1)}\left(\vec{p}^{-1} \mid \pi\right)^{\smile} s\left(x^{(1)}\left(\vec{p}^{-1} \mid \pi\right)+y^{(1)}\left(\vec{p}^{-1} \mid \pi\right), x^{(1)}\left(\vec{p}^{-1} \mid \pi\right)\right) \geq\right. \\
u\left((p-\epsilon) y(t)^{\smile} s(x(t)+y(t)), x\right)>u\left(p y^{\smile} s(x+y), x\right)
\end{aligned}
$$

for $t>0$ sufficiently small.

This contradicts that $\vec{p}$ is a BE equilibrium.

Hence, $(x, y)$ must be a competitive equilibrium strategy and $p$ a competitive equilibrium price. Q.E.D.

For $p>0$ such that $D(p)>0, \mu(p)=-\frac{p}{D(p)} D^{\prime}(p)$ denote the price elasticity of demand at price $p$.

If $D(p)$ is linear then by the Law of Demand, $\mu(p)$ increases as $p$ increases. 
The following is a non-existence result under palpable conditions. For the sake of simplicity we assume in what follows that $s=0$, so that costs of production if there be any are fixed costs.

THEOREM 6 Let u be continuously differentiable (i.e. $C^{1}$ ). Let $p$ be a competitive equilibrium price and suppose that $x>0$, where the pair $(x, y)$ solves

\section{Maximize $u(p \eta, \xi)$}

subject to $\xi+\eta \leq \bar{x}, p \eta \geq 0$ and $\xi, \eta \geq 0$.

Then the strategy profile $\vec{p}$ where each seller chooses $p$ as his strategy is not a BE equilibrium strategy. Thus if sellers consume positive quantities of the produced good at every competitive equilibrium, then a BE equilibrium does not exist.

Proof: We know from lemma 8, that $y$ (as in the statement of this theorem) $=\frac{D(p)}{H}$.

Assume the conditions of the theorem.

Then the pair $(x, y)$ solves

Maximize $u(p \eta, \xi)$

subject to $\xi+\eta \leq \bar{x}, \eta \leq \frac{D(p)}{H}, p \eta \geq 0$ and $\xi, \eta \geq 0$.

as well as

Maximize $u(p \eta, \xi)$

subject to $\xi+\eta \leq \bar{x}, \eta \leq \frac{D(p)}{H-1}, p \eta \geq 0$ and $\xi, \eta \geq 0$, since $\frac{D(p)}{H}<\frac{D(p)}{H-1}$.

No seller can do better by charging a lower price for if $p^{\prime}<p$, and there exists $(\xi, \eta)$ such that $\xi+\eta \leq \bar{x}, p^{\prime} \eta \geq 0, \xi, \eta \geq 0$, and $u\left(p^{\prime} \eta, \xi\right)>u(p y, x)$, then clearly $p \eta>p^{\prime} \eta \geq 0$ as well as $u(p \eta, \xi)>u(p y, x)$ contradicting our assumption about the pair $(x, y)$. Since no improvement is possible by unilateral deviation to a lower price, without incorporating the constraint $\eta \leq D\left(p^{\prime}\right)$, it is certainly the case that no improvement is possible by unilateral deviation to a lower price, if we add the constraint $\eta \leq D\left(p^{\prime}\right)$

Hence in order to consider unilateral deviations that lead to an improvement for a seller, it is enough to consider deviations that arise due to increasing the price. 
Consider seller 1 and suppose he raises his price from $p$ to $p+\epsilon$ for some $\epsilon>0$. Then the residual demand curve faced by seller 1 , is $D(p+\epsilon)^{\cup} \frac{(H-1)}{H} D(p)<\frac{D(p)}{H}$. Hence seller 1 will have to reduce his sales.

Since production is costless and his utility function is increasing, we might as well assume that if he cuts back his sales by $\delta \geq D(p)^{\smile} D(p+\epsilon)$, he increases his consumption of the produced good by $\delta$.

We need to show that $u((p+\epsilon)(y-\delta), x+\delta)-u(p y, x)>0$.

Let $\alpha=\frac{(H-1)}{H} y$ and $R_{\alpha}(y-\delta)=\left(\frac{D(p)}{H}-\delta\right) p(D(p)-\delta)$.

Consider the function $\delta \mid \rightarrow u\left(R_{\alpha}(y-\delta), x+\delta\right)$ with domain $\left(0, \frac{D(p)}{H}\right)$.

The derivative of this function is equal to $-M R_{\alpha}(y-\delta) D_{1} u\left(R_{\alpha}(y-\delta), x+\right.$ $\delta)+D_{2} u\left(R_{\alpha}(y-\delta), x+\delta\right)=-p(\alpha+y-\delta)\left[1-\frac{1}{\mu(p(\alpha+y-\delta))}\right] D_{1} u\left(R_{\alpha}(y-\delta), x+\right.$ $\delta)+D_{2} u\left(R_{\alpha}(y-\delta), x+\delta\right)=D_{2} u\left(R_{\alpha}(y-\delta), x+\delta\right)-p(\alpha+y-\delta) D_{1} u\left(R_{\alpha}(y-\right.$ $\delta), x+\delta)+\frac{p(\alpha+y-\delta)}{\mu(p(\alpha+y-\delta)} D_{1} u\left(R_{\alpha}(y-\delta), x+\delta\right)=p(\alpha+y-\delta) D_{1} u\left(R_{\alpha}(y-\delta), x+\right.$ $\delta)\left[\frac{1}{\mu(p(\alpha+y-\delta))}-\frac{p(\alpha+y-\delta) D_{1} u\left(R_{\alpha}(y-\delta), x+\delta\right)-D_{2} u\left(R_{\alpha}(y-\delta), x+\delta\right)}{p D_{1} u\left(R_{\alpha}(y-\delta), x+\delta\right)}\right]$.

Since $p$ is a competitive equilibrium price and since both $p y$ and $x$ are positive, by (iii) of Proposition $3, p D_{1} u\left(R_{\alpha}(y), x\right)-D_{2} u\left(R_{\alpha}(y), x\right)=0$. Thus $\frac{1}{\mu(p)}-$ $\frac{p D_{1} u\left(R_{\alpha}(y), x\right)-D_{2} u\left(R_{\alpha}(y), x\right)}{p D_{1} u\left(R_{\alpha}(y), x\right)}>0$. Thus for $\delta>0$ sufficiently small $\frac{1}{\mu(p(\alpha+y-\delta))}-$ $\frac{p(\alpha+y-\delta) D_{1} u\left(R_{\alpha}(y-\delta), x+\delta\right)-D_{2} u\left(R_{\alpha}(y-\delta), x+\delta\right)}{p D_{1} u\left(R_{\alpha}(y-\delta), x+\delta\right)}>0$.

Thus, for $\delta>0$ sufficiently small,

$$
\begin{gathered}
p(\alpha+y-\delta) D_{1} u\left(R_{\alpha}(y-\delta), x+\delta\right)\left[\frac{1}{\mu(p(\alpha+y-\delta))}-\right. \\
\left.\frac{p(\alpha+y-\delta) D_{1} u\left(R_{\alpha}(y-\delta), x+\delta\right)-D_{2} u\left(R_{\alpha}(y-\delta), x+\delta\right)}{p D_{1} u\left(R_{\alpha}(y-\delta), x+\delta\right)}\right]>0,
\end{gathered}
$$

and so $u\left(R_{\alpha}(y-\delta), x+\delta\right)>u(p y, x)$.

Let $\epsilon=p(\alpha+y-\delta)-p(\alpha+y-\delta)$.

Thus, $u\left((p+\epsilon) y^{(2)}\left(\vec{p}^{-1} \mid p+\epsilon\right), x^{(2)}\left(\vec{p}^{-1} \mid p+\epsilon\right)\right) \geq u\left(R_{\alpha}(y-\delta), x+\delta\right)>u(p y, x)$

This proves the theorem. Q.E.D.

Note that the demand function for the market replicated $k$ times is simply $k D(p)$. Thus each replica of a seller continues to face the same problem that the seller did in the un-replicated market, irrespective of the number of times we decide to replicate it. Hence all the results obtained above are independent 
of the number of sellers $H$, provided $H$ is no less than two.

Acknowledgement: I would like to thank Ludovic Julien for very helpful comments, especially about the inappropriateness of the title of an earlier version of this paper (A Simple Cournotian Model of Production and Trade). I would also like to thank Simone Tonin for helpful comments and bibliographic improvements. An earlier version of this paper was presented at a faculty seminar at the Chennai campus of the Institute for Financial Management and Research. I would like to thankall those who attended the seminar and in particular S. Maheswaran for searching questions about the paper. I would also like to thankJyoti Prasad Mukhopadhyayand Santosh Dash for their observations during the seminar and after. Subsequently this paper was presented at the International Conference of the Twenty Fifth Summer Festival on game theory where it received enlightening and / or encouraging comments from Rabah Amir, Bruno Badia, Debapriya Sen, Maryam Sami and Andrea Maria Vittorio Venturini for which I would like to thank them immensely. At the same venue Pradeep Dubey pointed out to me an alternative proof of the existence of generalized Nash equilibrium using Brouwer's fixed point theorem when the payoff functions are continuous and concave in the respective strategies and the constraint correspondences are upper semicontinuous. I would like to thank him for the proofof this result since this result is what is essentially used in the proofs of Theorems 1 and 3 in this paper. Errors that still remain are the sole responsibility of the author.

\section{References}

[1] Arrow, K.J. and G. Debreu : "Existence of equilibrium for a competitive economy", Econometrica, 22 (1954): 265-290.

[2] Beckmann, J. M. : "Edgeworth-Bertrand duopoly revisited", in R. Henn, ed., Operations Research Verfahren III, Meisenheim: Verlag Anton Hain (1965).

[3] Berge, C. :"Topological Spaces", Macmillan, New York (1963).

[4] Debreu, G. : "Theory of Value: An Axiomatic Analysis of Economic Equilibrium", Monograph 17, Cowles Foundation for Research in Economics, Yale University Press, New Haven (1959).

[5] Dahl, C. : "International Energy Markets: Understanding Pricing, Policies and Profits", Tulsa OK: Pennwell Press, March (2004).

[6] Dubey, P. and M. Shubik. : "A Closed Economic System with Production and Exchange Modeled as a Game of Strategy", Cowles Foundation Discussion Paper No. 429, New Haven, Connecticut (1976).

[7] Gabszewicz, J. J. and P. Michel : "Oligopoly equilibrium in exchange economies", in B.C. Eaton and R. G. Harris, (Eds.) Trade, technology and 
economics: Essays in honor of Richard G. Lipsey, Elgar, Cheltenham, UK (1997).

[8] Gabszewicz, J. J. and J.-P. Vial : "Oligopoly "A la Cournot" in a General Equilibrium Analysis", Journal of Economic Theory, 4 (1972): 381-400.

[9] Geanakoplos, J. : "Nash and Walras equilibrium via Brouwer", Economic Theory 21 (2003): 585-603.

[10] Lahiri, S.: "Asymptotic Convergence to competitive equilibrium of oligopoly equilibria", available at http://ssrn.com/abstract=1739486 (2010).

[11] Lahiri, S. : "Comparative statics of oligopoly equilibrium in a pure exchange economy", Modern Economy, 2 (2011): 77-83.

[12] Levitan, R., and M. Shubik : "Price duopoly and capacity constraints", International Economic Review 13 (1972): 111-122.

[13] Novshek, W. : "Perfectly competitive markets as the limits of Cournot markets", Journal of Economic Theory, 35 (1985): 72-82.

[14] Shapley, L.S. : "Non-cooperative general exchange", in S.A.Y. Lin (ed.), Theory of measurement of economic externalities, Academic Press, New York (1976).

[15] Shapley, L.S. and M. Shubik : "Trade using one commodity as a means of payment", Journal of Political Economy 85 (1977), 937-968.

[16] Shubik, M. : "Commodity money, oligopoly, credit and bankruptcy in a general equilibrium model", Western Economic Journal 11 (1973), 24-38.

[17] Tasnadi, A. : "Existence of Pure Strategy Nash Equilibrium in Bertrand Edgeworth Oligopolies", Economics Letters, 63 (1999): 201-206.

[18] Vives, X. : "Cournot and the oligopoly problem", European Economic Review, 33 (1989): 503-514.

[19] Vives, X. : "Oligopoly Pricing: Old Ideas and New Tools", The MIT Press, Cambridge, Massachusetts (1999). 